[Article]

\title{
人类蛋白激酶组的多靶点分子对接系统
}

\author{
邓玉玲 ${ }^{1}$ 余 䞦 $^{2}$ 黄 强 ${ }^{1, *}$ \\ ('复旦大学生命科学学院, 上海 200438; '2 中国科学院上海生命科学研究院生物化学与细胞生物学研究所, 上海 200031)
}

\begin{abstract}
摘要: 蛋白激酶在信号转导、基因转录和蛋白翻译等生物过程起关键性作用, 因而与大量人类疾病密切相 关。所以, 蛋白激酶的抑制剂笁选是抗肿瘤药物开发的热点, 正在向基于全激酶组的高通量多靶点筛选模式 发展。为了降低大规模实验篮选的成本, 提高成功率, 本文构建人类蛋白激酶组的多靶点分子对接系统, 对 抑制剂-激酶组的相互作用进行预测。我们首先利用同源模建方法, 对人类激酶组约 500 个激酶变异体的催化 域进行结构建模; 接着以催化域结构模型为受体, 用已知激酶抑制剂进行分子对接, 对抑制剂与各激酶变异 体的结合亲和力进行了定量计算。结果显示, 本文所建立的多靶点分子对接系统可以准确预测抑制剂与激酶 变异体的相互作用, 结合自由能的计算值与实验值有很强的相关性。所以, 该分子对接系统可用于多靶点激 酶抑制剂的计算笁选, 为激酶抑制剂开发与抗肿瘤药物设计提供理论依据。
\end{abstract}

关键词：蛋白激酶；激酶抑制剂；相互作用预测；同源模建；分子对接

中图分类号: 0641

\section{A Multi-Target Docking System of Human Kinome}

\author{
DENG Yu-Ling ${ }^{1} \quad$ YU Lu ${ }^{2} \quad$ HUANG Qiang ${ }^{1, *}$ \\ ('School of Life Sciences, Fudan University, Shanghai 200438, P. R. China; ' ${ }^{2}$ Institute of Biochemistry and Cell Biology, \\ Shanghai Institutes for Biological Sciences, Chinese Academy of Sciences, Shanghai 200031, P. R. China)
}

\begin{abstract}
Protein kinases play critical roles in many biological processes, including signal transduction, gene transcription, and protein translation, and are therefore closely associated with various disease states. The screening of kinase inhibitors has become an important aspect of anti-tumor drug development, and has been refined to allow high-throughput, multi-target screening based on the entire human kinome. To reduce the experimental costs of large-scale inhibitor screening and to increase the success rate, our group has designed a multi-target molecular docking system capable of predicting kinase-inhibitor interactions. In this work we initially used homology modeling to construct three-dimensional (3D) models for approximately 500 catalytic domains of human kinase variants. We subsequently performed molecular docking to calculate the binding affinities of kinase-inhibitor pairs, employing the 3D models as receptors and kinase inhibitors as ligands. The results show that our multi-target docking system accurately predicts the interactions between known inhibitors and kinase variants, and that the calculated binding affinities are highly correlated with the experimental values. Thus, this molecular docking system could be used for computational screening of multi-target kinase inhibitors, thereby providing a theoretical basis for the development of kinase inhibitors and the design of anti-tumor drugs.
\end{abstract}

Key Words: Protein kinase; Kinase inhibitor; Interaction prediction; Homology modeling; Molecular docking

Received: March 24, 2016; Revised: May 16, 2016; Published online: May 17, 2016.

*Corresponding author. Email: huangqiang@fudan.edu.cn; Tel: +86-21-51630589.

The project was supported by the National Natural Science Foundation of China (30570406, 91430112), National Hi-tech Research and Development Program of China (863) (2008AA02Z311), Shanghai Natural Science Foundation, China (13ZR1402400).

国家自然科学基金(30570406, 91430112), 国家高技术研究发展计划(863) (2008AA02Z311)及上海市自然科学基金(13ZR1402400)资助项目

(C) Editorial office of Acta Physico-Chimica Sinica 


\section{1 引言}

蛋白激酶 $(\mathrm{PK})$ 是一类催化蛋白质磷酸化的酶, 对生物体内 $1 / 3$ 以上的蛋白进行调控, 从而影响基 因转录、蛋白翻译、细胞分化和信号转导等众多 关键性生物进程 ${ }^{1}$ 。目前人类基因组中已有 518 种 蛋白激酶被确认, 占整个基因组的 $2 \%$ 左右 ${ }^{2}$ 。研究 表明, 蛋白激酶可作为药物靶点影响包括白血 病、心血管疾病、糖尿病和癌症等多种疾病的发 生 ${ }^{3-5}$, 已发现 400 多种以上的疾病与蛋白激酶存在 直接或间接的联系 ${ }^{6}$ 。因此, 以激酶为靶标进行抑 制剂的篎选一直为药物研发的热点。现已有不少 激酶抑制剂(PKI)应用于相关疾病的治疗, 并取得 了显著的成效 ${ }^{7}$, 如 Gleecec、Imatinib 和 Erlotinib 三种酪氨酸激酶抑制剂就被美国食品药物管理局 (FDA)批准用于抗肿瘤治疗 ${ }^{8}$ 。

通过对真核生物蛋白激酶的序列分析发现, 蛋白激酶都有一个由 250-300个氨基酸构成的催 化功能区。该功能区的氨基酸序列同源度高, 可 分为 12 个亚区。不同蛋白激酶的特异性主要由 VI、VIII、IX亚区的氨基酸序列决定, 如 VI 区序 列 Asp-Leu-Lys-Pro-Glu-Asn一般存在于丝氨酸或 苏氨酸激酶中, 而 Asp-Leu-Arg-Ala-Asn 或 AspLeu-Ala-Ala-Arg-Asn 多在酪氨酸激酶中发现 ${ }^{9}$ 。不 同蛋白激酶的催化功能区通过折叠形成相似的二 叶状结构 ${ }^{10-12}$ (图 1): 其中 N 端包含 ATP 针定所必 需的 P 环(p-loop), C 端含有调节激酶活性的活化环 (activation loop), 而催化蛋白质磷酸化的催化环 (catalytic loop)位于两叶相连的铰链区。这种催化 域结构具有三种重要功能: 介导 ATP 或 GTP 的结 合与定向; 介导底物蛋白或多肽的结合与定向; 催化 $\gamma$-磷酸基团从载体分子转移至底物蛋白的氨基

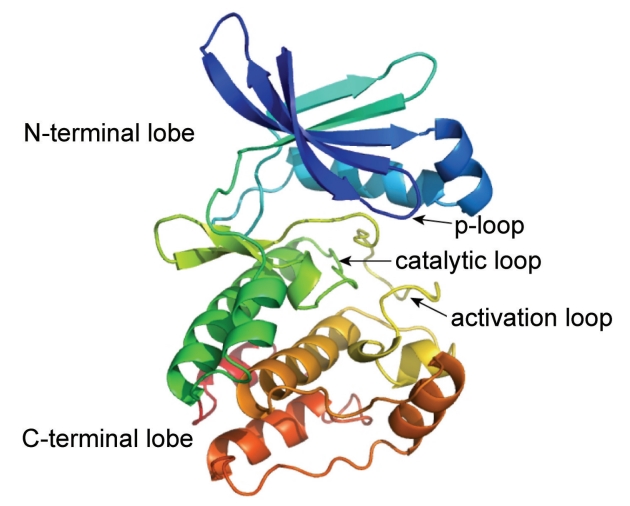

图 1 蛋白激酶催化域结构

Fig.1 Structure of protein kinase catalytic domain
酸残基上。正是由于催化域对蛋白激酶功能的实 现有重要的作用, 因此该结构成为激酶抑制剂开 发最为关注的对象。通常的激酶抑制剂主要就是 通过与 ATP 或肽底物竞争 ATP 结合位点或肽结合 位点等发挥作用的，抑制活性的高低依赖于抑制 剂对相关结合区域氨基酸残基的结合亲和力 ${ }^{13}$ 。

目前，蛋白激酶抑制剂的开发正由传统的只 针对单一激酶的线性篮选开发模式, 向基于全激 酶组的高通量并行篎选模式转变 ${ }^{14}$ 。大规模的高通 量并行䇥选有利于发现多靶点激酶抑制剂, 了解 抑制剂可能的脱靶效应。但是, 针对人类激酶组 500 多种激酶变异体进行大规模实验篮选是一个大 工程, 耗时且花费巨大, 因此有必要利用计算手 段先进行大规模的虚拟篮选, 为实验篎选提供理 论指导, 以降低大规模实验篮选的成本, 提高成 功率。虽然结构基因组学的发展为虚拟節选提供 了丰富的数据, 但是已知晶体结构的蛋白激酶只 占人类激酶组的一部分。为此, 本文从激酶催化 域的结构建模出发, 利用同源模建和大规模结构 精修的方法, 构建出了所有蛋白激酶变异体催化 域的高分辨率三维(3D)结构模型, 然后利用分子对 接方法对蛋白激酶-抑制剂的结合亲和性进行定量 计算, 为抑制剂的篮选提供理论指导。

\section{2 计算方法}

\section{1 蛋白激酶组催化域 3D 结构模型的构建}

\subsection{1 模型构建}

由于激酶组催化域有一定的保守性, 本文采 用同源模建(homology modeling) ${ }^{15}$ 的方法进行催化 域结构建模。本文从 PDB (Protein Data Bank)数据 库 ${ }^{16}$ 下载了约 110 个人蛋白激酶的晶体结构 (见 Supporting Information 表 S1), 并对这些晶体结构 的催化域进行精修: 对结构中缺失的原子坐标进 行补齐; 缺失残基数大于 4 的缺失片断用 Rosetta 程序 ${ }^{17}$ 的 loop refinement 算法重建, 类似方法可参 见我们的前期工作 ${ }^{18}$ 。最后, 利用 Rosetta程序的 relax 算法对上述处理过的结构进行优化, 选取能 量最低且结构合理的 $3 \mathrm{D}$ 模型为建模模板。

建模流程(图 2)包括: 首先对全激酶组进行多 重序列比对，篮选出与特定目标序列同源度最高 的模板; 然后利用 Modeller 程序 ${ }^{15}$ 对目标激酶的催 化域进行同源建模; 最后利用 Rosetta程序的 relax 算法对所建模型进行大规模的全原子模型结构优 


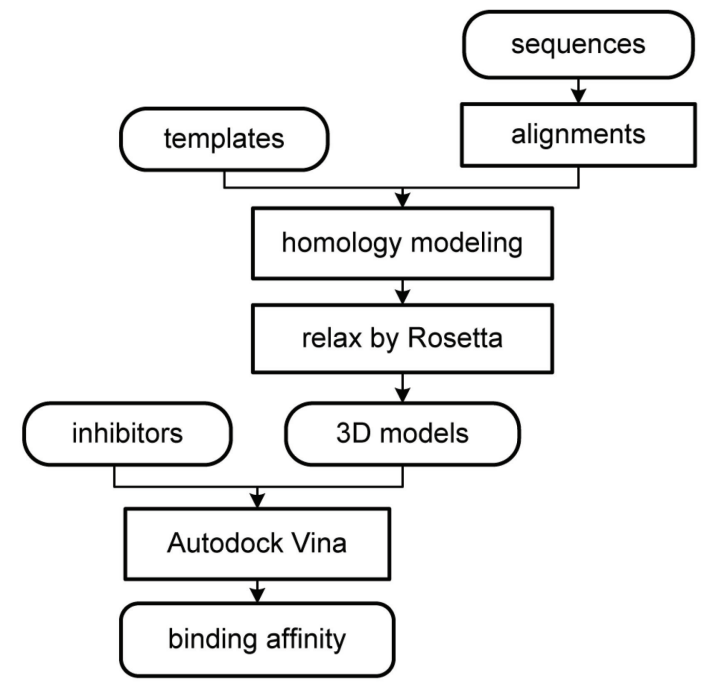

图 2 人类蛋白激酶组的多靶点分子对接系统

Fig.2 Flowchart for the multi-target docking system of human kinome

化, 由此获得的能量最低的构象就是后续分子对 接用的受体结构模型。上述序列比对为 Modeller 软 件系统产生, 同源建模和 Rosetta 优化的参数均采 用系统默认。

\section{1 .2 模型可靠性检验}

为检验所开发的全激酶组催化域建模流程的 可靠性, 本文主要采用以下方法进行验证：针对 从 PDB 数据库下载的 113 个人蛋白激酶, 分别在排 除自身晶体结构作为模板的情况下，利用上述建 模流程进行模型构建, 最后将预测模型与晶体结 构进行比对。

本文主要采用了 TM-align 算法 ${ }^{19}$ 计算的 $\mathrm{C}_{a^{-}}$ RMSD (均方根偏差) 和 TM-score 值, 及 LGA (localglobal alignment) 程序 ${ }^{20}$ 分析所得的 GDT (global distance test) 值, 作为对预测模型质量的定量评 估。 $\mathrm{C}_{a}-\mathrm{RMSD}$ 是比较结构相似性最常用的参数, 若晶体结构与预测模型的 $\mathrm{C}_{a}-\mathrm{RMSD}$ 值在 $3.0 \mathrm{~nm}$ 以 内, 则认为预测模型是可信的 ${ }^{21}$ 。但是, 局部位点 的过度偏移可能导致 RMSD 偏差过大。因此, 本 文将 TM-align 算法所提出的、可以对结构整体的 相似度进行良好描述的 TM-score 值也纳入了评价 系统。TM-score 值的范围在 0-1之间, 值越大表 明两个结构的相似度越高。经统计, 高质量的全 原子模型 TM-score 值在 0.5 以上 ${ }^{22}$ 。GDT 值是集中 于结构整体概况的另一个参数, 它是指预测结构 与目标结构相对应的 $\mathrm{C}_{\alpha}$ 原子距离, 在某个特定的 阈值之内的残基比例, 常用GDT_TS (total score of
GDT) 和距离条件更为严苛的 GDT_HA (high accuracy of GDT)来描述。GDT 值越大，表明两个 结构越相似。GDT_TS 值大于 60 的模型被认为是 可靠的模型 23 。

除上述参数外, 本文还使用常规的 Procheck ${ }^{24}$ 和 Verify $3 \mathrm{D}^{25}$ 程序进行了辅助验证。Procheck 可以 提供处于核心区 (most favoured regions)、允许区 (additional allowed regions)、大致允许区(generously allowed regions)和禁阻区(disallowed regions)的氨 基酸残基占总数的百分比。一般来说，前三个区 域的残基百分比之和(本文用 Procheck-precent表示) 大于 $95 \%$ 则模型的质量很好。Verify $3 \mathrm{D}$ 主要是比 较模型与氨基酸一级结构的关系，当在 3D/1D 图 谱中的分值大于等于 0.2 的氨基酸百分比(本文用 Verify3d-precent 表示)高于 $80 \%$ 时，即可获得 PASS 评价, 证明模型质量是可靠的。

\section{2 抑制剂-蛋白激酶组的分子对接}

AutoDock 程序 ${ }^{26}$ 可用于计算配体-受体对接的 最低能量构象和对应的结合自由能, 是预测配体 结合能力的有效手段，被广泛应用于抑制剂虚拟 筛选系统。本文采用 AutoDock Vina 程序 ${ }^{27}$ 进行分 子对接(molecular docking)。在对接过程中, 本文 将晶格的中心(center)坐标设为 $(0,0,0)$, 大小 (size) 设为 $(25,25,25)$, 能量偏差 (energy range) 设为 $12.56 \mathrm{~kJ} \cdot \mathrm{mol}^{-1}$, 其余参数则为系统默认，该程序 计算的结合自由能即为本文衡量抑制剂的蛋白激 酶结合能力的依据。

为了验证所构建预测系统的可靠性, 本文采用 以下方法进行验证：首先，从 Pubchem Compound 数据库 ${ }^{28}$ 下载了约 40 种已进行临床试验的小分子抑 制剂, 并进行了相应的能量优化, 作为分子对接 的配体; 然后, 将这些小分子抑制剂与 2.1.1 节获 取的激酶组的全部催化域 3D 结构模型进行分子对 接, 篮选出能量最低的构象并记录其所对应的结 合自由能; 最后，根据从文献 ${ }^{29}$ 中收集的这些小分 子抑制剂与对应的 270 多种激酶受体的解离常数实 验值 (见 Supporting Information 表 S2), 由公式 $\Delta G_{\text {bind }}=R T \ln K_{\mathrm{d}}$ (其中 $\Delta G_{\text {bind }}$ 为结合自由能, $K_{\mathrm{d}}$ 是抑 制剂与激酶结合的解离常数, $R$ 取 $34.81 \mathrm{~kJ} \cdot \mathrm{mol}^{-1}$. $\mathrm{K}^{-1}, T$ 取 $\left.298.15 \mathrm{~K}\right)^{30}$ 计算出实验结合自由能, 通过 比较结合自由能计算值与实验值之间的偏差来判 断预测系统是否可靠。 


\section{3 结果与讨论}

\section{1 催化域建模流程的可靠性}

本文运用 2.1 节的建模方法获得了人类蛋白激 酶组的 518 个激酶变异体催化域的 3D 结构模型, 组成了人蛋白激酶-抑制剂分子对接的受体库。我 们采用 2.1.2节描述的方法检验所开发的全激酶组 催化域 3D 建模流程的可靠性, 即针对已经晶体结 构的 113 个激酶, 排除自身晶体结构作为建模模 板, 利用建模流程进行 3D 模型构建, 并把预测模 型与晶体结构进行比较。表 1 统计了已知晶体结构 的人蛋白激酶的 $\mathrm{C}_{a}-\mathrm{RMSD}$ 值的区间分布, 表中数 据表示在对应区间内的模型个数。约有 $78 \%$ (88/ 113) 的预测模型与原晶体结构比较的 $\mathrm{C}_{\alpha}-\mathrm{RMSD}$ 值 在 $3.0 \mathrm{~nm}$ 以下, 说明此激酶组建模系统有较高的 可信性。同源建模网站 Swiss-MODEL ${ }^{31}$ 类似的可靠 性检验所得的比例是 $46 \%$, 这也进一步说明建模 流程的可靠性。此外, 结果中有 10 种激酶的 $\mathrm{C}_{a^{-}}$ RMSD 在 $1.5 \mathrm{~nm}$ 以下, 显示了很好的建模效果。 而 $\mathrm{C}_{\alpha}-\mathrm{RMSD}$ 值大于 $3.0 \mathrm{~nm}$ 的 26 种激酶中，有 10 种 与模板之间的序列相似度小于 $40 \%$, 这与同源模 建算法在目标序列与模板的同源性较低时有较大 局限性的情况是一致的。至于其中 2 种序列相似度 高于 $90 \%$ 的激酶, 其 $\mathrm{C}_{\alpha}-\mathrm{RMSD}$ 值较大的原因可能是 预测结构局部氨基酸位点的偏移较大, 为此, 我们 还采用其它参量(如 TM-score、GDT_TS) 及 Procheck、Verify_3D对其模建结构进行评估。

图 3(a,b) 描述了分属于各激酶组 (CAMK、 CMGC、AGC、STE、TKL、CK1、TK及 Other)的 这 113 种蛋白激酶对应的模板同源性与 $\mathrm{C}_{a}-\mathrm{RMSD}$ 、

\section{表 1 人类蛋白激酶预测模型的 $\mathrm{C}_{a}-\mathrm{RMSD}$ 区间分布}

Table 1 C $\mathrm{C}_{a}$-RMSD distribution for predicted models of human protein kinases

\begin{tabular}{crrrrc}
\hline \multirow{2}{*}{ Identity $/ \%{ }^{\text {a }}$ Model l } & \multicolumn{5}{c}{ Distribution $^{\mathrm{b}}$} \\
\cline { 3 - 6 } & & $0-1.5 \mathrm{~nm}$ & $1.5-3.0 \mathrm{~nm}$ & $3.0-4.0 \mathrm{~nm}$ & $4.0-5.0 \mathrm{~nm}$ \\
\hline $10-30$ & 14 & 1 & 8 & 4 & 1 \\
$30-40$ & 19 & 1 & 13 & 5 & 0 \\
$40-50$ & 19 & 1 & 11 & 7 & 0 \\
$50-60$ & 17 & 0 & 16 & 1 & 0 \\
$60-70$ & 9 & 3 & 5 & 1 & 0 \\
$70-80$ & 11 & 0 & 8 & 3 & 0 \\
$80-90$ & 15 & 3 & 11 & 1 & 0 \\
$\geq 90$ & 9 & 1 & 6 & 2 & 0 \\
all & 113 & 10 & 78 & 25 & 1 \\
\hline
\end{tabular}

RMSD: root mean square deviation. ${ }^{a}$ sequence identity interval; ${ }^{b}$ number of predicted models in this $\mathrm{C}_{\alpha}-\mathrm{RMSD}$ interval
TM-score 的关联。在表 1 中显示的唯一 $\mathrm{C}_{a}$-RMSD 值大于 $4.0 \mathrm{~nm}$ 的激酶为 CMGC 组的 SRPK(PDB code: $\left.1 \mathrm{WBP}^{32}\right)$, 模型分辨率不高的原因可能是其 与最佳模板的序列相似性仅为 $17.3 \%$, 没有达到同 源建模的正常标准 ${ }^{33}$, 但其 TM-score 值为 0.62 , 说 明其整体相似度仍高于 0.5 的建模平均精度。

从各激酶组的结果分析也可以看出本文的建 模流程具有较高的可靠性。以 CAMK组为例, 全 部 20 种激酶有 16 种模型(约 $80 \%$ ) 的 $\mathrm{C}_{\alpha}-\mathrm{RMSD}$ 值在 $3.0 \mathrm{~nm}$ 以下, 其中两种高分辨率模型 CaMK2a (PDB code: 2VZ6 ${ }^{34}$ ) 和 CaMK2d (PDB code: $\left.2 \mathrm{VN} 9^{34}\right)$ 的 $\mathrm{C}_{a}-\mathrm{RMSD}$ 值小于 $1.5 \mathrm{~nm}$ 。对于 $\mathrm{C}_{a}$-RMSD 值大于 $3.0 \mathrm{~nm}$ 的 4 种激酶 CHK1 (PDB code: 1IA8 ${ }^{35}$ )、 CHK2 (PDB code: 2CN5 ${ }^{36}$ )、 MAPKAPK2 (PDB code: 1KWP ${ }^{37}$ ) 和 MNK1 (PDB code: $\left.2 \mathrm{HW} 6{ }^{38}\right)$, 其 TM-score 分布在 $0.65-0.80$ 之 间, 说明整体相似性是不错的, 它们与模板的同源 性在 30\%-40\%, 在同源模建可能导致的误差之内。

从 TM-score 的角度同样可以看出本文建模方 法的优越性。在所有结果中仅有 AGC 组的 GPRK6 (PDB code: $2 \mathrm{ACX}^{39}$ ) 和 TK 组的 ABL1(PDB code: $1 \mathrm{OPL}{ }^{40}$ ) 两种激酶的 TM-score 小于 0.5 , 分析发 现, 这两种激酶的 $\mathrm{C}_{a}$-RMSD 在 $0.3 \mathrm{~nm}$ 左右, 且与 模板的序列同源性在 $40 \%$ 左右, 说明本文的结果 具有一致性。

图 3(c,d) 显示了LGA 分析获得的 GDT_TS 和 GDT HA 值与对应的模板同源性的关联。从图 3c 可以看出, 本文构建的极大部分预测模型的 GDT_TS 值都大于 60 , 且其中相当一部分结构的 GDT_TS 值高于 80 , 再次说明了建模系统可靠 性。对于 GDT_TS 值未达到 60 的预测模型, 可以 发现除序列同源性小于 $20 \%$ 的两个模型，即 CMGC 组的 SRPK1 (PDB code: $1 \mathrm{WBP}^{32}$ ) 和 Other 组的 Haspin (PDB code: $2 \mathrm{VUW}^{41}$ ) 外, 其余预测模 型的 GDT_TS 位于 50-60之间, 仍属于可靠性高 的模型。从图 $3 \mathrm{~d}$ 可见, 即便对预测模型和目标结 构的对应 $\mathrm{C}_{\alpha}$ 原子的距离条件更为严苛 $(0.5 、 1 、 2$ 和 $4 \mathrm{~nm}$ ), 仍有一定数量的预测模型的 GDT_HA 值高 于 60 , 也说明了本文的建模方法在构建高精度模 型方面有很大的潜力。

图 3(e, f) 分别为 Procheck 和 Verify_3D 程序对 预测模型质量进行验证的结果。由图 $3 \mathrm{e}$ 可见, 大 部分预测模型的氨基酸残基落在核心区、允许区 

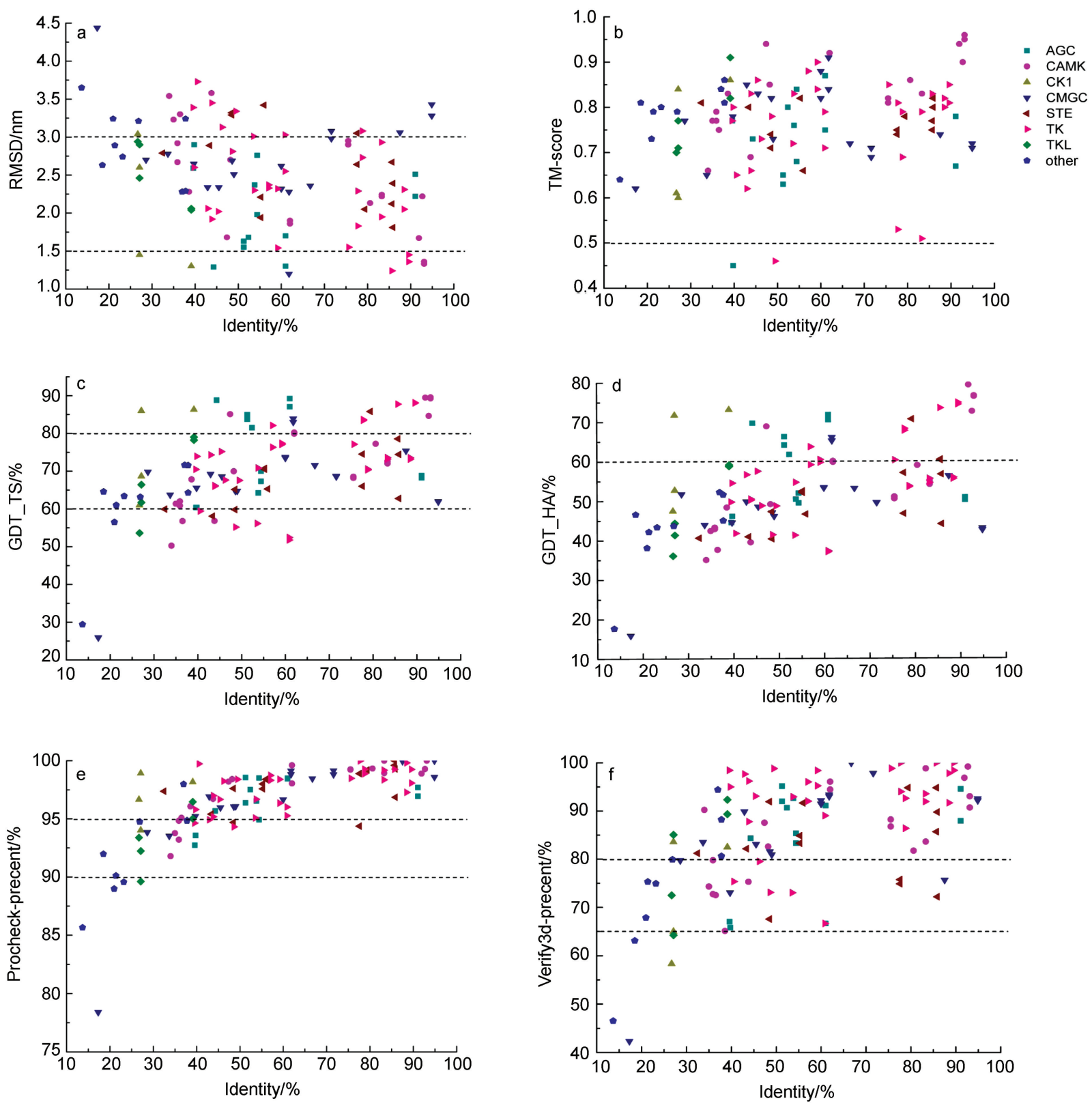

图 3 各蛋白激酶组预测模型验证结果

Fig.3 Validation results of predicted models for human kinase groups (a) $\mathrm{C}_{\alpha}$-RMSD; (b) TM-score; (c) GDT_TS; (d) GDT_HA; (e) Procheck-precent; (f) Verify3d-precent $v s$ identity

以及大致允许区的比例之和是大于 $95 \%$ 的标准 的, 说明这些预测模型的结构是可靠的。未达到 95\%标准的模型中，除了前面我们已经分析的个别 模型外，其余也是处于 $90 \%-95 \%$ 的区间内，说明 这些模型的质量仍是不错的。同样，从 Verify_3D 的结果(图 3f)也可以发现, 本文建模流程构建的绝 大部分模型的质量都是可靠的，即高于 $80 \%$ 的标 准。特别地, 在表 1 显示的序列相似度高于 $90 \%$ 但 $\mathrm{C}_{\alpha}-\mathrm{RMSD}$ 值大于 $0.3 \mathrm{~nm}$ 的两种激酶, 经 Procheck 和 Verify_3D程序验证的结果均为可靠。综上本文
的建模流程具有较高的可靠性。

根据本文的建模流程所构建的模型中，部分 激酶与模板的序列相似性并不显著, 如 AGC 组中 PKACa (PDB code： 2GU8 ${ }^{42}$ ) 仅为 $44.3 \%$, 但其预 测结构与晶体结构之间的一致性从 $\mathrm{C}_{\alpha}$-RMSD (1.29 $\mathrm{nm}) 、$ TM-score (0.73) 以及 GDT_TS (88.824)、 GDT_HA (69.705)等各方面得到了验证。图 4a显示 了 PKACa 的预测结构 (PKACa_model) 与晶体结构 (PKACa)的比对情况。由图可见, 该激酶催化域的 各部位都得到了良好的匹配。图 4b 标示了该激酶 

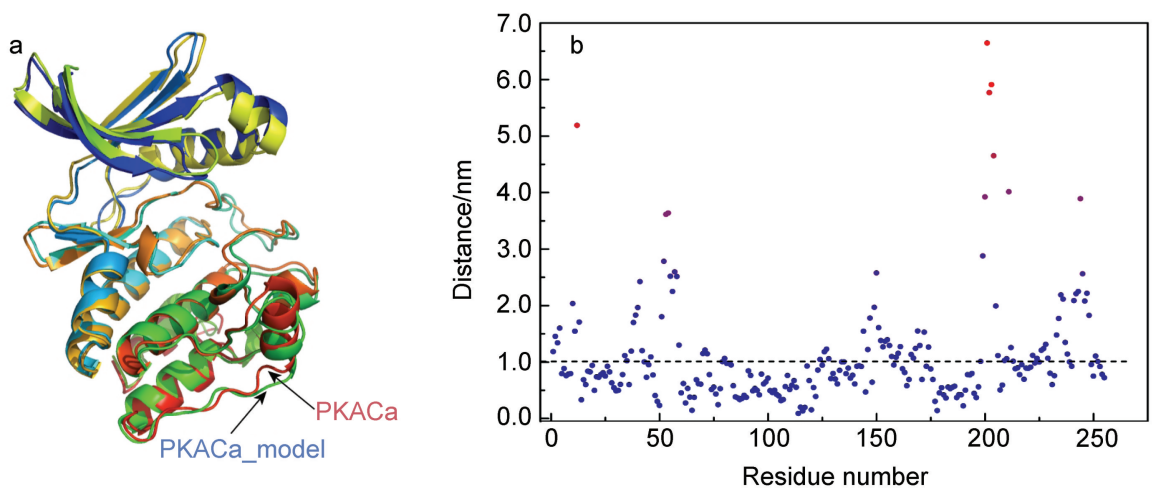

图 4 PKACa 激酶的预测模型与晶体结构的比较

Fig.4 Comparison between predicted model and crystal structure of PKACa kinase

(a) structural alignment; (b) distance of each $\mathrm{C}_{a}$ atom pair

预测结构与晶体结构对应位置的 $\mathrm{C}_{\alpha}$ 原子之间的距 离, 从中可以看出, 除个别位点有较大偏移外, 结构中大部分位点的距离都在 $1.0 \mathrm{~nm}$ 以下, 印证 了预测结构整体与晶体结构的匹配度高。

\section{2 抑制剂-蛋白激酶的分子对接}

\subsection{1 预测系统可靠性验证}

表 2 归纳了本文人类蛋白激酶组-抑制剂分子 对接系统的可靠性验证结果。鉴于已有研究表 明，对两个不同测试集，AutoDock Vina 计算的结 合自由能的偏差分别为 11.51 和 $11.93 \mathrm{~kJ} \cdot \mathrm{mol}^{-1}{ }^{27}$, 本文采用 $11.30 \mathrm{~kJ} \cdot \mathrm{mol}^{-1}$ 为计算可靠性的验证标 准。由表可见, 各激酶组的自由能偏差值 $(\Delta \Delta G=$ $\left.\left|\Delta G_{\text {exp }}-\Delta G_{\text {cal }}\right|\right)$ 小于 $11.30 \mathrm{~kJ} \cdot \mathrm{mol}^{-1}$ 的比例均高于 $80 \%$ 。尤其是 AGC、CAMK 以及 TKL 三组, 其比 例均高于 $90 \%$ 。所有组别的自由能偏差值达到标 准的平均比例为 $88 \%$, 且平均标准差仅为 $7.26 \mathrm{~kJ}$. $\mathrm{mol}^{-1}$ 。这些结果都表明本文的计算系统具有较高 的预测精度。

图 5a 显示了 CAMK 激酶组中的 344 对激酶-抑
制剂的结合自由能的计算值与实验值的分布图。 根据本文的标准，较好的预测应分布在对角线两 侧距离为 $11.30 \mathrm{~kJ} \cdot \mathrm{mol}^{-1}$ 的平行线范围内, 越靠近 对角线的点表明预测的精度越高, 对角线上的点 说明计算值与实验值精确匹配。从图中可以看 出, 该激酶组中极大部分点落在预示结果良好的 平行线范围内, 甚至有一部分点显示出精确匹配。

\subsection{2 抑制剂-蛋白激酶相互作用对的篇选}

有实际价值的抑制剂与特定激酶的结合能力 往往较强, 即结合自由能或解离常数 $K_{\mathrm{d}}$ 值位于一 定的阈值范围内。通常抑制剂解离常数要求在 $\mu \mathrm{mol} \cdot \mathrm{L}^{-1}$ 级别 ${ }^{43}$, 因此本文选取了 $K_{\mathrm{d}}=1 \mu \mathrm{mol} \cdot \mathrm{L}^{-1}$ 的阈值进行抑制剂-蛋白激酶相互作用对的篎选, 并与实验结果进行比较, 获取了本文预测系统的 预测准确率。

節选结果与实验数据比较得到表 3 。由表可 见，在 $K_{\mathrm{d}}$ 阈值为 $1 \mu \mathrm{mol} \cdot \mathrm{L}^{-1}$ 的情况下，预测系统的 平均预测准确率高达 $56.3 \%$, 且所有激酶组的预测 准确率均高于 $50 \%$, 表明本文的预测系统具有很

表 2 多靶点分子对接系统的验证结果

Table 2 Prediction performance of the multi-target docking system

\begin{tabular}{|c|c|c|c|c|c|}
\hline \multirow{2}{*}{ Kinase group } & \multirow{2}{*}{ Interaction $^{\mathrm{a}}$} & \multirow{2}{*}{ Standard error } & \multicolumn{3}{|c|}{ Percentage $(\%)$ of $\Delta \Delta G^{\mathrm{b}}$} \\
\hline & & & $0-4.19 \mathrm{~kJ} \cdot \mathrm{mol}^{-1}$ & $4.19-11.30 \mathrm{~kJ} \cdot \mathrm{mol}^{-1}$ & $>11.30 \mathrm{~kJ} \cdot \mathrm{mol}^{-1}$ \\
\hline AGC & 146 & 6.32 & 48.6 & 44.5 & 6.8 \\
\hline CAMK & 344 & 6.95 & 48.8 & 41.6 & 9.6 \\
\hline $\mathrm{CK} 1+$ other & 284 & 7.20 & 48.6 & 38.8 & 12.7 \\
\hline CMGC & 240 & 7.70 & 37.1 & 48.3 & 14.6 \\
\hline STE & 241 & 8.16 & 44.4 & 40.2 & 15.4 \\
\hline $\mathrm{TK}$ & 943 & 8.12 & 46.0 & 38.0 & 16.0 \\
\hline TKL & 184 & 6.36 & 51.6 & 40.8 & 7.6 \\
\hline average $^{c}$ & & 7.26 & 46.4 & 41.7 & 11.8 \\
\hline
\end{tabular}

anumber of predicted kinase-inhibitor interactions; ${ }^{b}$ percentage of $\Delta \Delta G=\left|\Delta G_{\text {exp }}-\Delta G_{\text {cal }}\right|$ in this interval; ' $a v e r a g e$ values of all the kinase groups 

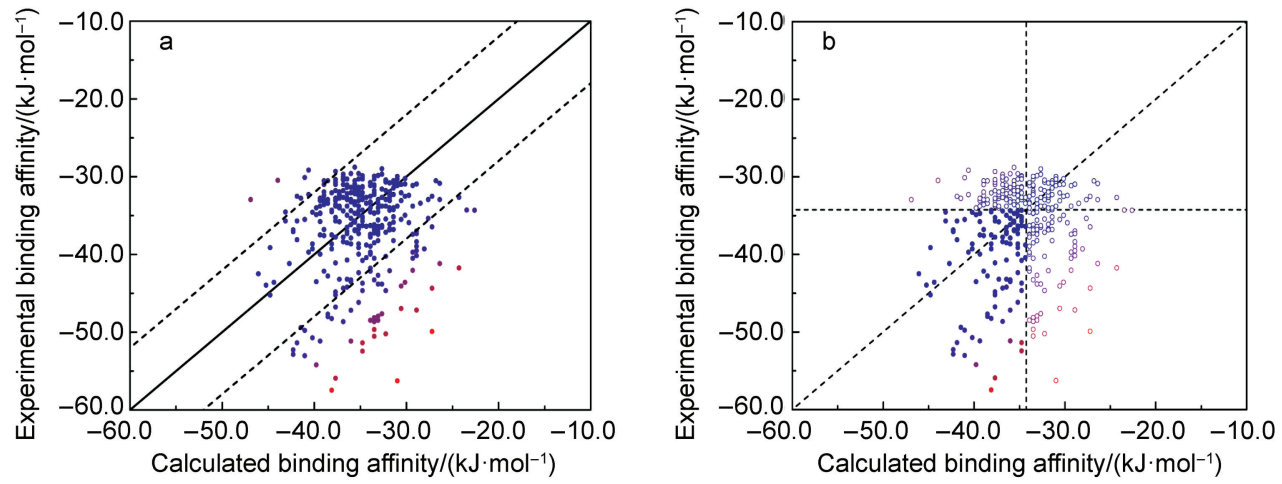

图 5 CAMK激酶组的分子对接结果

Fig.5 Results of molecular docking for CAMK kinase group

(a) correlation between calculated and experimental binding affinities; (b) predicted kinase-inhibitor interaction pairs selected by $K_{\mathrm{d}}<1 \mu \mathrm{mol} \cdot \mathrm{L}^{-1}$

表 3 多靶点对接系统的篮选结果验证

Table 3 Hit percentages of our multi-target docking system

\begin{tabular}{ccc}
\hline Kinase group & Selected number $^{\mathrm{a}}$ & ${\text { Hit } / \%^{\mathrm{b}}}^{\mathrm{b}}$ \\
\hline AGC & 120 & 50.8 \\
CAMK & 199 & 55.3 \\
CK1 + Other & 126 & 57.1 \\
CMGC & 136 & 63.2 \\
STE & 64 & 57.8 \\
TK & 512 & 58.4 \\
TKL & 132 & 51.5 \\
\hline
\end{tabular}

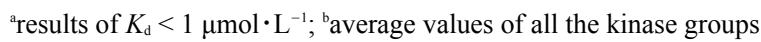

好的预测效果。具体以 CAMK 激酶组为例 (图 $5 b$ ), 当 $K_{\mathrm{d}}$ 小于 $1 \mu \mathrm{mol} \cdot \mathrm{L}^{-1}$ 可计算 $\Delta G_{\text {bind }}$ 小于 -34.25 $\mathrm{kJ} \cdot \mathrm{mol}^{-1}$, 所以通过计算模型篮选出的相互作用对 在图中表示为坚虚线 $\left(\Delta G_{\text {bind }}=-34.25 \mathrm{~kJ} \cdot \mathrm{mol}^{-1}\right)$ 左侧 的点，而实验 $K_{\mathrm{d}}$ 值真正小于 $1 \mu \mathrm{mol} \cdot \mathrm{L}^{-1}$ 的相互作用 对位于横虚线的下侧, 重叠区域的点(实心的点)即 为运用本文的预测系统预测准确的相互作用对, 预测准确率为 $55.3 \%$ 。

图 6(a,b) 分别展示了 CAMK 激酶组的模型 PIM3 与 LY-333531 抑制剂、MELK 与 Imatinib 抑制 剂的结合情况。PIM3 与 LY-333531 抑制剂的计算 结合自由能为 $-44.80 \mathrm{~kJ} \cdot \mathrm{mol}^{-1}$, 实验值为 -45.18 $\mathrm{kJ} \cdot \mathrm{mol}^{-1}$ ，偏差仅为 $0.38 \mathrm{~kJ} \cdot \mathrm{mol}^{-1}$, 在图 $5 \mathrm{~b}$ 中显示为 实心的点, 即在 $K_{\mathrm{d}}$ 阈值为 $1 \mu \mathrm{mol} \cdot \mathrm{L}^{-1}$ 时, 本文系统 计算值与实验值相吻合的结果。MELK 与 Imatinib 抑制剂的计算结合自由能为 $-34.33 \mathrm{~kJ} \cdot \mathrm{mol}^{-1}$, 实验 值为 $-32.66 \mathrm{~kJ} \cdot \mathrm{mol}^{-1}$ ，两者偏差在标准误差 11.30 $\mathrm{kJ} \cdot \mathrm{mol}^{-1}$ 内, 在图 $5 \mathrm{~b}$ 中显示为虚线左侧的点, 即本 文系统计算篮选出的相互作用对，鉴于 Imatinib 抑 制剂已被FDA 批准用于抗肿瘤治疗，说明其已是 被认可的一种激酶抑制剂，而用我们的模型与对
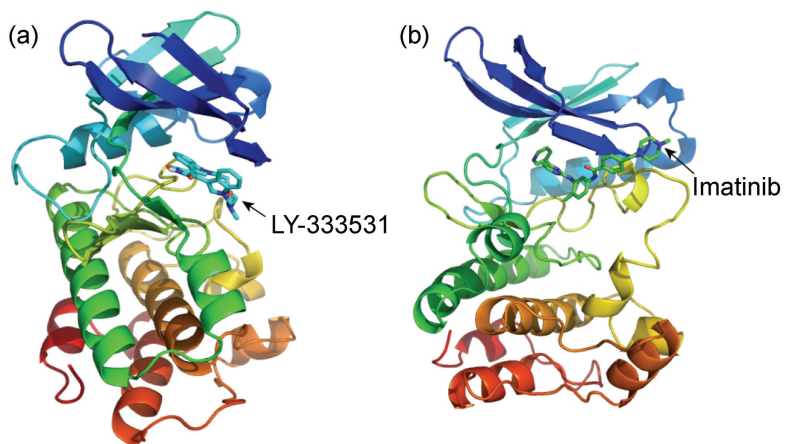

图 6 CAMK激酶组的分子对接模型

Fig.6 Molecular docking models of CAMK kinase group

(a) binding mode of PIM3 and LY-333531; (b) binding mode of MELK and Imatinib

接方法，通过计算其与各蛋白激酶的结合相互作 用，可以准确预测其与对应激酶受体的结合亲和 性。这也说明了本文系统可以作为激酶抑制剂的 篮选手段，当对一系列小分子抑制剂进行计算配 体一激酶相互作用篮选，可以预测出某个或某几个 激酶的抑制剂分子。而且从图 6 可以看出, LY333531 抑制剂主要作用在 PIM3 激酶的催化环和活 化环区域; Imatinib 抑制剂主要作用在 MELK激酶 的 P环区域，所以本文的预测系统预测也可用于抑 制剂-激酶的结合模式的挖掘。

\section{4 结 论}

目前，蛋白激酶的抑制剂節选正在向基于全 激酶组的高通量多靶点簰选模式发展, 为了降低 大规模实验篮选的成本，提高成功率，本文构建 人类蛋白激酶组的多靶点分子对接系统, 以对抑 制剂一激酶组的相互作用进行预测。该系统首先利 用 PDB 数据库提供的人蛋白激酶晶体结构催化域 
为模板, 通过同源模建、模型优化等步骤, 获取 了人蛋白激酶组 500 多个激酶变异体的催化域 3D 结构模型。通过选取的 $\mathrm{C}_{\alpha}$-RMSD、TM-score 和 GDT 等多个参数, 以及 Procheck 和 Verify_3D 程序 对建模流程的可靠性进行评估, 结果表明所开发 的 3D 建模流程具有较高的可靠性。然后, 以所得 的催化域 $3 \mathrm{D}$ 结构模型为受体, 用已知激酶抑制剂 进行分子对接, 对抑制剂与各激酶变异体的结合 亲和力进行了定量计算。结果显示, 所建立的分 子对接系统可以准确预测抑制剂与激酶变异体的 相互作用, 结合自由能的计算值与实验值有很强 的相关性。因此, 本文所构建的分子对接系统可 用于多靶点激酶抑制剂的计算䇻选, 从而为激酶 抑制剂开发与抗肿瘤药物设计提供理论依据。

致 谢: 感谢复旦大学生命科学学院黄强教授实验室的袁 慧同学对全文文字进行了仔细的修改。

Supporting Information: The crystal structures of human protein kinases as the templates of modeling and experimental $K_{\mathrm{d}}$ values for 38 small molecular inhibitors against corresponding kinase receptors have been included. This information is available free of charge via the internet at http://www.whxb.pku.edu.cn.

\section{References}

(1) Hunter, T. Cell 2000, 100, 113. doi: 10.1016/S0092-8674(00) 81688-8

(2) Noble, M. E. M. Science 2004, 303, 1800. doi: 10.1126/ science. 1095920

(3) Bild, A. H.; Yao, G.; Chang, J. T.; Wang, Q.; Potti, A.; Chasse, D.; Joshi, M.; Harpole, D.; Lancaster, J. M.; Berchuck, A.; Olson, J. A.; Marks, J. R.; Dressman, H. K.; West, M.; Nevins, J. R. Nature 2005, 439, 353. doi: 10.1038/nature04296

(4) Cohen, P. Nat. Rev. Drug Discov. 2002, 4, 309. doi: 10.1038/ $\operatorname{nrd} 773$

(5) Manning, G.; Whyte, D. B.; Martinez, R.; Hunter, T.; Sudarsanam, S. Science 2002, 298, 1912. doi: 10.1126/ science. 1075762

(6) Weinmann, H.; Metternich, R. ChemBioChem 2005, 6, 455. doi: 10.1002/cbic. 200500034

(7) Vieth, M.; Sutherland, J. J.; Robertson, D. H.; Campbell, R. M. Drug Discov. Today 2005, 10, 839. doi: 10.1016/s1359-6446(05) 03477-X

(8) Wu, P.; Nielsen, T. E.; Clausen, M. H. Trends Pharmacol. Sci. 2015, 7, 422. doi: 10.1016/j.tips.2015.04.005

(9) Scheeff, E.; Bourne, P. PLoS Comput. Biol. 2005, 1, e49. doi: 10.1371/journal.pcbi.0010049.eor

(10) Krauss, G. Biochemistry of Signal Transduction and Regulation, 3rd ed; WILEY-VCH Verlag GmbH \& Co. KGaA: Weinheim, 2004; p 269.

(11) Zheng, J.; Knighton, D. R.; Eyck, L. F. T.; Karlsson, R.; Xuong, N.; Taylor, S. S.; Sowadski, J. M. Biochemistry 1993, 2154. doi: 10.1021/bi00060a005

(12) Wei, L.; Hubbard, S. R.; Smith, R. F.; Ellis, L. Curr. Opin. Struc. Biol. 1994, 4, 450. doi: 10.1016/s0959-440x(94)90115-5

(13) Dancey, J.; Sausville, E. A. Nat. Rev. Drug Discov. 2003, 2, 296. doi: $10.1038 / \operatorname{nrd} 1066$

(14) Goldstein, D. M.; Gray, N. S.; Zarrinkar, P. P. Nat. Rev. Drug Discov. 2008, 7, 391. doi: 10.1038/nrd2541

(15) Fiser, A. Methods Mol. Biol. 2010, 673, 73. doi: 10.1007/978-160761-842-3_6

(16) Berman, H. M. Acta Crystallogr. A 2008, 64, 88. doi: 10.1107/ S0108767307035623

(17) Das, R.; Baker, D. Annu. Rev. Biochem. 2008, 77, 363. doi: 10.1146/annurev.biochem.77.062906.171838

(18) Xu, M.; Yu, L.; Wan, B.; Yu, L.; Huang, Q. PloS One 2011, 7, e22644. doi: 10.1371/journal.pone.0022644.

(19) Zhang, Y; Skolnick, J. Nucleic Acids Res. 2005, 33, 2302. doi: 10.1093/nar/gki524

(20) Zemla, A. Nucleic Acids Res. 2003, 31, 3370. doi: 10.1093/nar/ gkg571

(21) Zhang, Y. Curr. Opin. Struct. Biol. 2008, 18, 342. doi: 10.1016/j. sbi.2008.02.004

(22) Zhang, Y.; Skolnick, J. Proteins 2004, 57, 702. doi: 10.1002/ prot. 20264

(23) Kryshtafovych, A.; Monastyrskyy, B.; Fidelis, K. Proteins 2014, 82, 7. doi: 10.1002/prot.24399

(24) Laskowski, R. A.; MacArthur, M. W.; Mass, D. S.; Thornton, J. M. J. Appl. Crystallogr. 1993, 26, 283. doi: 10.1107/ S0021889892009944

(25) Eisenberg, D.; Lüthy, R.; Bowie, J. Method Enzymol. 1997, 6364, 396. doi: 10.1016/S0076-6879(97)77022-8

(26) Park, H.; Lee, J.; Lee, S. Proteins 2006, 65, 549. doi: 10.1002/ prot. 21183

(27) Trott, O.; Olson, A. J. J. Comput. Chem. 2009, 2, 455. doi: $10.1002 /$ jcc. 21334

(28) Wang, Y.; Xiao, J.; Suzek, T. O.; Zhang, J.; Wang, J.; Bryant, S. H. Nucleic Acids Res. 2009, 37, W623. doi: 10.1093/nar/gkp456

(29) Karaman, M. W.; Herrgard, S.; Treiber, D. K.; Gallant, P.; Atteridge, C. E.; Campbell, B. T.; Chan, K. W.; Ciceri, P.; Davis, M. I.; Edeen, P. T.; Faraoni, R.; Floyd, M.; Hunt, J. P.; Lockhart, D. J.; Milanov, Z. V.; Morrison, M. J.; Pallares, G.; Patel, H. K.; Pritchard, S.; Wodicka, L. M.; Zarrinkar, P. P. Nat. Biotechnol. 2008, 26, 127. doi: 10.1038/nbt1358

(30) Huey, R.; Morris, G. M.; Olson, A. J.; Goodsell, D. S. J. Comput. Chem. 2007, 6, 1145. doi: 10.1002/jcc.20634

(31) Biasini, M.; Bienert, S.; Waterhouse, A.; Arnold, K.; Studer, G.; Schmidt, T.; Kiefer, F.; Cassarino, T. G.; Bertoni, M.; Bordoli, L.; Schwede, T. Nucleic Acids Res. 2014, 42, W252. 
doi: 10.1093/nar/gku340

(32) Ngo, J. C. K.; Chakrabarti, S.; Ding, J.; Velazquez-Dones, A.; Nolen, B.; Aubol, B. E.; Adams, J. A.; Fu, X.; Ghosh, G. Mol. Cell 2005, 20, 77. doi: 10.1016/j.molcel.2005.08.025

(33) Kryshtafovych, A.; Fidelis, K. Drug Discov. Today 2009, 14, 386. doi: 10.1016/j.drudis.2008.11.010

(34) Rellos, P.; Pike, A. C. W.; Niesen, F. H.; Salah, E.; Lee, W. H.; von Delft, F.; Knapp, S. PLoS Biol. 2010, 7, 426. doi: 10.1371/ journal.pbio. 1000426

(35) Chen, P.; Luo, C.; Deng, Y.; Ryan, K.; Register, J.; Margosiak, S.; Tempczyk-Russell, A.; Nguyen, B.; Myers, P.; Lundgren, K.; Kan, C.; Connor, P. M. O. Cell 2000, 6, 681. doi: 10.1016/ S0092-8674(00)80704-7

(36) Oliver, A. W.; Paul, A.; Boxall, K. J.; Barrie, S. E.; Aherne, G. W.; Garrett, M. D.; Mittnacht, S.; Pearl, L. H. EMBO J. 2006, 25, 3179. doi: 10.1038/sj.emboj.7601209

(37) Meng, W. J. Biol. Chem. 2002, 277, 37401. doi: 10.1074/jbc.
C200418200

(38) Jauch, R.; Cho, M.; Jakel, S.; Netter, C.; Schreiter, K.; Aicher, B.; Zweckstetter, M.; Jackle, H.; Wahl, M. C. EMBO J. 2006, 25, 4020. doi: 10.1038/sj.emboj.7601285

(39) Elkins, J. M.; Amos, A.; Niesen, F. H.; Pike, A. C. W.; Fedorov, O.; Knapp, S. Protein Sci. 2009, 4, 782. doi: 10.1002/pro.82

(40) Nagar, B.; Hantschel, O.; Young, M. A.; Scheffzek, K.; Veach, D.; Bornmann, W.; Clarkson, B.; Superti-Furga, G.; Kuriyan, J. Cell 2003, 6, 859. doi: 10.1016/S0092-8674(03)00194-6

(41) Knoechel, T. R.; Tucker, A. D.; Robinson, C. M.; Phillips, C.; Taylor, W.; Bungay, P. J.; Kasten, S. A.; Roche, T. E.; Brown, D. G. Biochemistry 2006, 45, 402. doi: 10.1021/bi051402s

(42) Biondi, R. M.; Komander, D.; Thomas, C. C.; Lizcano, J. M.; Maria, D.; Alessi, D. R.; Aalten, D. M. F. EMBO J. 2002, 21, 4219. doi: 10.1093/emboj/cdf437

(43) Knight, Z. A.; Shokat, K. M. Chem. Biol. 2005, 12, 621. doi: 10.1016/j.chembiol.2005.04.011 
Supporting Information for Acta Phys. -Chim. Sin. 2016, 32 (9), 2355-2363

doi: $\quad$ 10.3866/PKU.WHXB201605171

\title{
人类蛋白激酶组的多靶点分子对接系统
}

\author{
邓玉玲 ${ }^{1}$ 余 璐 ${ }^{2}$ 黄 强 ${ }^{1, *}$ \\ ( ${ }^{1}$ 复旦大学生命科学学院, 上海 $200438 ;{ }^{2}$ 中国科学院上海生命科学研究院生物化学与细胞生物学研究 \\ 所, 上海 200031)
}

\section{A Multi-Target Docking System of Human Kinome}

\author{
DENG Yu-Ling ${ }^{1} \quad$ YU Lu $^{2} \quad$ HUANG Qiang ${ }^{1, *}$ \\ ( ${ }^{1}$ School of Life Sciences, Fudan University, Shanghai 200438, P. R. China; $\quad{ }^{2}$ Institute of Biochemistry and Cell \\ Biology, Shanghai Institutes for Biological Sciences, Chinese Academy of Sciences, Shanghai 200031, P. R. \\ China)
}

*Corresponding author. Email: huangqiang@fudan.edu.cn; Tel: +86-21-51630589. 
表 S1 人蛋白激酶模板的晶体结构

Table S1 Crystal structures of human protein kinases as the templates of modeling

\begin{tabular}{|c|c|c|c|}
\hline Kinase group & Kinase & PDB code & Resolution/nm \\
\hline \multirow[t]{13}{*}{ AGC } & AKT1 & $3 C Q U$ & 2.2 \\
\hline & AKT2 & $1 \mathrm{GZK}$ & 2.3 \\
\hline & DMPK1 & 2VD5 & 1.85 \\
\hline & GPRK6 & $2 A C X$ & 2.7 \\
\hline & MSK1 & $1 \mathrm{VZO}$ & 2.6 \\
\hline & PDK1 & $1 \mathrm{H} 1 \mathrm{~W}$ & 1.8 \\
\hline & $\mathrm{PKCa}$ & 2GU8 & 2 \\
\hline & $\mathrm{PKCb}$ & $210 \mathrm{E}$ & 2.2 \\
\hline & $\mathrm{PKCi}$ & $1 Z R Z$ & 2.6 \\
\hline & PKCt & 1XJD & 3 \\
\hline & ROCK1 & 2ETR & 2 \\
\hline & RSK1 & $2 Z 7 Q$ & 2.6 \\
\hline & SGK1 & 2R5T & 2 \\
\hline \multirow[t]{20}{*}{ CAMK } & AMPKa2 & $2 \mathrm{H} 6 \mathrm{D}$ & 1.85 \\
\hline & CaMK1d & $2 \mathrm{JC6}$ & 2.3 \\
\hline & CaMK1g & 2JAM & 1.7 \\
\hline & CaMK2a & 2VZ6 & 2.3 \\
\hline & CaMK2b & 3BHH & 2.5 \\
\hline & CaMK2d & 2VN9 & 2.3 \\
\hline & CaMK2g & $2 \mathrm{~V} 7 \mathrm{O}$ & 2.25 \\
\hline & CASK & $3 \mathrm{COG}$ & 2.19 \\
\hline & $\mathrm{CHK} 1$ & $1 \mathrm{IA} 8$ & 1.7 \\
\hline & $\mathrm{CHK} 2$ & 2CN5 & 2.25 \\
\hline & DAPK1 & $1 \mid G 1$ & 1.8 \\
\hline & DAPK2 & $1 \mathrm{ZWS}$ & 2.9 \\
\hline & DAPK3 & 2J90 & 2 \\
\hline & MAPKAPK2 & $1 \mathrm{KWP}$ & 2.8 \\
\hline & MARK3 & $2 \mathrm{QNJ}$ & 2.79 \\
\hline & MNK1 & $2 \mathrm{HW} 6$ & 2.5 \\
\hline & PASK & 3DLS & 2.3 \\
\hline & PIM1 & $1 \mathrm{XWS}$ & 1.8 \\
\hline & PIM2 & $2|W|$ & 2.8 \\
\hline & TTN & $1 \mathrm{TKI}$ & 1.7 \\
\hline \multirow[t]{4}{*}{ CK1 } & CK1g2 & $2 \mathrm{C} 47$ & 2.4 \\
\hline & CK1g3 & $2 \mathrm{CHL}$ & 1.95 \\
\hline & VRK2 & $2 \mathrm{~V} 62$ & 1.7 \\
\hline & VRK3 & 2JII & 2 \\
\hline \multirow[t]{3}{*}{ CMGC } & CDK2 & $1 \mathrm{HCK}$ & 1.9 \\
\hline & CDK5 & $1 \mathrm{H} 4 \mathrm{~L}$ & 2.65 \\
\hline & CDK6 & $1 \mathrm{BI} 7$ & 3.4 \\
\hline
\end{tabular}




\begin{tabular}{|c|c|c|c|}
\hline & CDK7 & 1UA2 & 3.02 \\
\hline & CDK9 & 3BLR & 2.8 \\
\hline & CLK1 & $1 Z 57$ & 1.7 \\
\hline & CLK3 & 2EU9 & 1.53 \\
\hline & DYRK1A & $2 \mathrm{VX} 3$ & 2.4 \\
\hline & Erk2 & 1PME & 2 \\
\hline & Erk3 & $216 \mathrm{~L}$ & 2.25 \\
\hline & GSK3B & 1109 & 2.7 \\
\hline & JNK1 & $1 \mathrm{UKH}$ & 2.35 \\
\hline & JNK2 & $3 E 70$ & 2.14 \\
\hline & JNK3 & 1JNK & 2.3 \\
\hline & p38a & 1WFC & 2.5 \\
\hline & p38d & $3 \mathrm{COI}$ & 2.09 \\
\hline & p38g & 1CM8 & 2.4 \\
\hline & SRPK1 & 1WBP & 2.4 \\
\hline \multirow[t]{14}{*}{ STE } & MAP3K5 & $2 \mathrm{CLQ}$ & 2.3 \\
\hline & LOK & $2 \mathrm{~J} 7 \mathrm{~T}$ & 2 \\
\hline & MAP2K1 & $1 S 9 J$ & 2.26 \\
\hline & MAP2K2 & 1S9I & 2.45 \\
\hline & MAP2K6 & 3FME & 2.4 \\
\hline & MAP2K7 & 2DYL & 3.2 \\
\hline & MST1 & $3 \mathrm{COM}$ & 2.2 \\
\hline & MST3 & $3 \mathrm{CKW}$ & 1.96 \\
\hline & OSR1 & 2VWI & 2.15 \\
\hline & PAK1 & 1F3M & 2.3 \\
\hline & PAK4 & 2BVA & 2.3 \\
\hline & PAK5 & $2 \mathrm{~F} 57$ & 1.8 \\
\hline & PAK6 & $2 \mathrm{C} 30$ & 1.6 \\
\hline & SLK & $2 J 51$ & 2.1 \\
\hline \multirow[t]{15}{*}{ TK } & ABL1 & $10 P L$ & 3.42 \\
\hline & ACK & $1 U 46$ & 2 \\
\hline & BTK & $1 \mathrm{~K} 2 \mathrm{P}$ & 2.1 \\
\hline & CSK & $1 \mathrm{BYG}$ & 2.4 \\
\hline & EGFR & $1 \mathrm{M} 14$ & 2.6 \\
\hline & EphA2 & $1 \mathrm{MQB}$ & 2.3 \\
\hline & EphA3 & $2 \mathrm{GSF}$ & 1.77 \\
\hline & EPHA5 & 2R2P & 2.4 \\
\hline & EPHA7 & 2REI & 1.6 \\
\hline & ErbB4 & 3BBT & 2.8 \\
\hline & FES & 3BKB & 1.9 \\
\hline & FGFR1 & 1FGK & 2.4 \\
\hline & FGFR2 & $1 \mathrm{GJO}$ & 2.4 \\
\hline & FLT3 & 1RJB & 2.1 \\
\hline & FMS & $211 \mathrm{M}$ & 1.8 \\
\hline
\end{tabular}




\begin{tabular}{|c|c|c|c|}
\hline & FYN & 2DQ7 & 2.8 \\
\hline & $\mathrm{HCK}$ & $1 \mathrm{AD} 5$ & 2.6 \\
\hline & INSR & $1 \mathrm{IRK}$ & 1.9 \\
\hline & ITK & 1SM2 & 2.3 \\
\hline & JAK2 & $2 \mathrm{~B} 7 \mathrm{~A}$ & 2 \\
\hline & JAK3 & 1YVJ & 2.55 \\
\hline & KDR & 1VR2 & 2.4 \\
\hline & KIT & $1 P K G$ & 2.9 \\
\hline & LCK & 3LCK & 1.7 \\
\hline & MER & $2 \mathrm{P} 0 \mathrm{C}$ & 2.4 \\
\hline & MET & 1ROP & 1.8 \\
\hline & PYK2 & $3 \mathrm{cc} 6$ & 1.6 \\
\hline & $\mathrm{RET}$ & 2IVS & 2 \\
\hline & SRC & 1FMK & 1.5 \\
\hline & TIE2 & 1FVR & 2.2 \\
\hline \multirow[t]{5}{*}{ TKL } & ACTR2B & $2 \mathrm{QLU}$ & 2 \\
\hline & BRAF & $1 \mathrm{UWH}$ & 2.95 \\
\hline & IRAK4 & $2 N R U$ & 2 \\
\hline & TAK1 & $2 \mathrm{EVA}$ & 2 \\
\hline & TGFbR1 & $1 \mathrm{~B} 6 \mathrm{C}$ & 2.6 \\
\hline \multirow[t]{9}{*}{ Other } & AurA & $1 \mathrm{MUO}$ & 1.86 \\
\hline & CK2a1 & 1JWH & 1.9 \\
\hline & Haspin & 2VUW & 2.2 \\
\hline & MPSK1 & 2BUJ & 2.6 \\
\hline & NEK2 & 2JAV & 2.9 \\
\hline & PLK1 & 2OWB & 3.1 \\
\hline & PLK4 & $3 \mathrm{COK}$ & 1.8 \\
\hline & TTK & 3CEK & 2.6 \\
\hline & Wee1 & $1 \times 8 B$ & 2.2 \\
\hline
\end{tabular}


表 S2 38 种小分子抑制剂与对应激酶受体的解离常数 $\left(K_{d}\right)$ 实验值

Table S2 Experimental dissociation constant $\left(K_{\mathrm{d}}\right)$ values for 38 small molecular inhibitors against corresponding kinase receptors

\begin{tabular}{|c|c|c|c|c|c|}
\hline Inhibitor & Kinase & $K_{\mathrm{d}} /\left(\mathrm{nmol} \cdot \mathrm{L}^{-1}\right)$ & Inhibitor & Kinase & $K_{\mathrm{d}} /\left(\mathrm{nmol} \cdot \mathrm{L}^{-1}\right)$ \\
\hline ABT-869 & ALK & 1600 & LY-333531 & PKACa & 5400 \\
\hline ABT-869 & AurA & 1600 & LY-333531 & PKACb & 3200 \\
\hline ABT-869 & AurB & 390 & LY-333531 & PKCd & 3.6 \\
\hline ABT-869 & AurC & 71 & LY-333531 & PKCe & 11 \\
\hline ABT-869 & $A X L$ & 340 & LY-333531 & PKCh & 1800 \\
\hline ABT-869 & BMPR2 & 7800 & LY-333531 & PKCt & 2.5 \\
\hline ABT-869 & CDK11 & 74 & LY-333531 & PKD2 & 890 \\
\hline ABT-869 & CDK7 & 2900 & LY-333531 & PKG2 & 3100 \\
\hline ABT-869 & CDK8 & 95 & LY-333531 & PKN1 & 350 \\
\hline ABT-869 & CDK9 & 3000 & LY-333531 & PKN2 & 1500 \\
\hline ABT-869 & CLK1 & 8900 & LY-333531 & PLK4 & 4700 \\
\hline ABT-869 & CLK4 & 1600 & LY-333531 & RIOK1 & 810 \\
\hline ABT-869 & DDR1 & 58 & LY-333531 & SLK & 400 \\
\hline ABT-869 & DDR2 & 3800 & LY-333531 & smMLCK & 290 \\
\hline ABT-869 & EphA3 & 3500 & LY-333531 & SNRK & 320 \\
\hline ABT-869 & EphA6 & 330 & LY-333531 & STK33 & 3100 \\
\hline ABT-869 & EphA7 & 110 & MLN-518 & ALK2 & 2500 \\
\hline ABT-869 & EphB3 & 1400 & MLN-518 & AurC & 1600 \\
\hline ABT-869 & EphB4 & 1400 & MLN-518 & CLK1 & 630 \\
\hline ABT-869 & Erk7 & 2800 & MLN-518 & CLK4 & 1600 \\
\hline ABT-869 & FLT1 & 7.5 & MLN-518 & DDR1 & 1400 \\
\hline ABT-869 & FLT3 & 0.63 & MLN-518 & EGFR & 410 \\
\hline ABT-869 & FLT4 & 16 & MLN-518 & FLT1 & 3100 \\
\hline ABT-869 & FMS & 3.4 & MLN-518 & FLT3 & 3 \\
\hline ABT-869 & FRK & 400 & MLN-518 & FMS & 4.9 \\
\hline ABT-869 & HPK1 & 830 & MLN-518 & IRAK3 & 730 \\
\hline ABT-869 & IRR & 3900 & MLN-518 & KHS1 & 780 \\
\hline ABT-869 & JNK2 & 240 & MLN-518 & $\mathrm{KIT}$ & 2.7 \\
\hline ABT-869 & KDR & 8.1 & MLN-518 & PCTAIRE1 & 6000 \\
\hline ABT-869 & KHS1 & 910 & MLN-518 & PDGFRa & 2.4 \\
\hline ABT-869 & KHS2 & 3200 & MLN-518 & PDGFRb & 4.5 \\
\hline ABT-869 & $\mathrm{KIT}$ & 2 & MLN-518 & TRKA & 450 \\
\hline ABT-869 & LATS2 & 1700 & MLN-518 & TRKB & 740 \\
\hline ABT-869 & LIMK2 & 4700 & MLN-518 & TRKC & 2000 \\
\hline ABT-869 & LOK & 460 & MLN-8054 & $\mathrm{ABL} 1$ & 820 \\
\hline ABT-869 & LTK & 550 & MLN-8054 & ABL2 & 3300 \\
\hline ABT-869 & MER & 870 & MLN-8054 & AurA & 6.5 \\
\hline ABT-869 & MET & 1300 & MLN-8054 & AurB & 43 \\
\hline ABT-869 & MNK1 & 4900 & MLN-8054 & AurC & 26 \\
\hline ABT-869 & MNK2 & 270 & MLN-8054 & $A X L$ & 440 \\
\hline
\end{tabular}




\begin{tabular}{|c|c|c|c|c|c|}
\hline ABT-869 & MST1 & 1400 & MLN-8054 & BLK & 68 \\
\hline ABT-869 & MST2 & 1500 & MLN-8054 & BMX & 3100 \\
\hline ABT-869 & MUSK & 10 & MLN-8054 & BTK & 4200 \\
\hline ABT-869 & PDGFRa & 4.2 & MLN-8054 & CLK2 & 3200 \\
\hline ABT-869 & PDGFRb & 1.9 & MLN-8054 & CLK3 & 2300 \\
\hline ABT-869 & PITSLRE & 1300 & MLN-8054 & CSK & 9000 \\
\hline ABT-869 & PITSLRE2 & 1200 & MLN-8054 & DDR1 & 4200 \\
\hline ABT-869 & PKACa & 6900 & MLN-8054 & DRAK1 & 190 \\
\hline ABT-869 & PLK3 & 8500 & MLN-8054 & DRAK2 & 8.1 \\
\hline ABT-869 & RET & 100 & MLN-8054 & EphA1 & 1800 \\
\hline ABT-869 & RIPK1 & 2800 & MLN-8054 & EphA2 & 370 \\
\hline ABT-869 & SLK & 450 & MLN-8054 & EphA3 & 2100 \\
\hline ABT-869 & STK33 & 3800 & MLN-8054 & EphA4 & 420 \\
\hline ABT-869 & TIE1 & 110 & MLN-8054 & EphA5 & 1400 \\
\hline ABT-869 & TIE2 & 450 & MLN-8054 & EphA6 & 580 \\
\hline ABT-869 & TRKA & 3100 & MLN-8054 & EphA7 & 1000 \\
\hline ABT-869 & TRKB & 1100 & MLN-8054 & EphB1 & 330 \\
\hline ABT-869 & TRKC & 210 & MLN-8054 & EphB3 & 3500 \\
\hline ABT-869 & YANK2 & 270 & MLN-8054 & EphB4 & 560 \\
\hline AMG-706 & ABL1 & 3900 & MLN-8054 & FER & 4400 \\
\hline AMG-706 & AurC & 1900 & MLN-8054 & FGFR1 & 2400 \\
\hline AMG-706 & BLK & 2200 & MLN-8054 & FGFR2 & 1700 \\
\hline AMG-706 & BRAF & 630 & MLN-8054 & FGFR3 & 1200 \\
\hline AMG-706 & BRK & 1000 & MLN-8054 & FGR & 220 \\
\hline AMG-706 & CRIK & 300 & MLN-8054 & FLT1 & 1000 \\
\hline AMG-706 & DDR1 & 260 & MLN-8054 & FLT4 & 3100 \\
\hline AMG-706 & EGFR & 15 & MLN-8054 & FYN & 1300 \\
\hline AMG-706 & EphA6 & 490 & MLN-8054 & $\mathrm{HCK}$ & 1500 \\
\hline AMG-706 & FGFR1 & 6200 & MLN-8054 & IRAK3 & 1400 \\
\hline AMG-706 & FGFR4 & 6800 & MLN-8054 & LCK & 590 \\
\hline AMG-706 & FGR & 6900 & MLN-8054 & LOK & 3700 \\
\hline AMG-706 & FLT1 & 12 & MLN-8054 & LYN & 1400 \\
\hline AMG-706 & FLT3 & 71 & MLN-8054 & MER & 730 \\
\hline AMG-706 & FLT4 & 9.7 & MLN-8054 & PLK4 & 410 \\
\hline AMG-706 & FMS & 5.6 & MLN-8054 & PYK2 & 2000 \\
\hline AMG-706 & FRK & 99 & MLN-8054 & ROS & 3100 \\
\hline AMG-706 & FYN & 2800 & MLN-8054 & SIK & 1700 \\
\hline AMG-706 & GCN2 & 4400 & MLN-8054 & SRC & 800 \\
\hline AMG-706 & $\mathrm{HH} 498$ & 220 & MLN-8054 & TEC & 730 \\
\hline AMG-706 & KDR & 26 & MLN-8054 & TIE1 & 1600 \\
\hline AMG-706 & $\mathrm{KIT}$ & 3.7 & MLN-8054 & TIE2 & 300 \\
\hline AMG-706 & LCK & 360 & MLN-8054 & TNK1 & 2400 \\
\hline AMG-706 & LOK & 1100 & MLN-8054 & TYRO3 & 3100 \\
\hline AMG-706 & LYN & 160 & MLN-8054 & YES & 260 \\
\hline
\end{tabular}




\begin{tabular}{|c|c|c|c|c|c|}
\hline AMG-706 & PDGFRa & 10 & PI-103 & BRAF & 2900 \\
\hline AMG-706 & PDGFRb & 9.1 & $\mathrm{PI}-103$ & CLK1 & 3900 \\
\hline AMG-706 & RAF1 & 880 & $\mathrm{PI}-103$ & DAPK1 & 2400 \\
\hline AMG-706 & RET & 14 & $\mathrm{PI}-103$ & DAPK2 & 2700 \\
\hline AMG-706 & SLK & 1300 & PI-103 & DAPK3 & 840 \\
\hline AMG-706 & ZAK & 8 & PI-103 & LIMK2 & 3500 \\
\hline AST-487 & ABL1 & 5.6 & $\mathrm{PI}-103$ & MRCKa & 1800 \\
\hline AST-487 & ABL2 & 3.4 & $\mathrm{PI}-103$ & PIK3CA & 1.5 \\
\hline AST-487 & ALK & 1400 & $\mathrm{PI}-103$ & RAF1 & 3700 \\
\hline AST-487 & AMPKa1 & 1400 & PI-103 & smMLCK & 1500 \\
\hline AST-487 & AurC & 740 & PKC-412 & AAK1 & 48 \\
\hline AST-487 & $A X L$ & 570 & PKC-412 & ACK & 120 \\
\hline AST-487 & BLK & 30 & PKC-412 & AKT1 & 950 \\
\hline AST-487 & BRAF & 1700 & PKC-412 & AKT2 & 780 \\
\hline AST-487 & CaMK1a & 3000 & PKC-412 & ALK & 270 \\
\hline AST-487 & CaMK4 & 3700 & PKC-412 & AMPKa1 & 180 \\
\hline AST-487 & CaMKK1 & 220 & PKC-412 & AMPKa2 & 460 \\
\hline AST-487 & CaMKK2 & 220 & PKC-412 & AurA & 120 \\
\hline AST-487 & CDK11 & 1.5 & PKC-412 & AurB & 62 \\
\hline AST-487 & CDK2 & 760 & PKC-412 & AurC & 170 \\
\hline AST-487 & CDK3 & 82 & PKC-412 & $A X L$ & 620 \\
\hline AST-487 & CDK5 & 1000 & PKC-412 & BIKE & 220 \\
\hline AST-487 & CDK7 & 4.5 & PKC-412 & BLK & 500 \\
\hline AST-487 & CDK8 & 1.4 & PKC-412 & BRSK1 & 1200 \\
\hline AST-487 & CDK9 & 190 & PKC-412 & BRSK2 & 650 \\
\hline AST-487 & CK1d & 2500 & PKC-412 & CaMK1a & 2000 \\
\hline AST-487 & CK1e & 4000 & PKC-412 & CaMK1d & 670 \\
\hline AST-487 & CK1g2 & 950 & PKC-412 & CaMK1g & 1800 \\
\hline AST-487 & CK1g3 & 97 & PKC-412 & CaMK2a & 20 \\
\hline AST-487 & CLK1 & 110 & PKC-412 & CaMK2b & 210 \\
\hline AST-487 & CLK2 & 1800 & PKC-412 & CaMK2d & 36 \\
\hline AST-487 & CLK4 & 290 & PKC-412 & CaMK2g & 140 \\
\hline AST-487 & CRIK & 52 & PKC-412 & CaMKK1 & 130 \\
\hline AST-487 & CSK & 2300 & PKC-412 & CaMKK2 & 73 \\
\hline AST-487 & DAPK3 & 3200 & PKC-412 & CDK11 & 7200 \\
\hline AST-487 & DDR1 & 0.69 & PKC-412 & CHK1 & 1300 \\
\hline AST-487 & DDR2 & 11 & PKC-412 & CK2a1 & 250 \\
\hline AST-487 & DLK & 2500 & PKC-412 & CLK1 & 350 \\
\hline AST-487 & EGFR & 520 & PKC-412 & CLK2 & 860 \\
\hline AST-487 & EphA2 & 160 & PKC-412 & CLK4 & 410 \\
\hline AST-487 & EphA3 & 80 & PKC-412 & CSK & 8700 \\
\hline AST-487 & EphA4 & 400 & PKC-412 & DAPK1 & 4700 \\
\hline AST-487 & EphA5 & 140 & PKC-412 & DAPK2 & 890 \\
\hline AST-487 & EphA6 & 380 & PKC-412 & DAPK3 & 1800 \\
\hline
\end{tabular}




\begin{tabular}{|c|c|c|c|c|c|}
\hline AST-487 & EphA7 & 1100 & PKC-412 & DDR1 & 7200 \\
\hline AST-487 & EphA8 & 18 & PKC-412 & DMPK1 & 3800 \\
\hline AST-487 & EphB1 & 180 & PKC-412 & DRAK1 & 1900 \\
\hline AST-487 & EphB2 & 130 & PKC-412 & DRAK2 & 4700 \\
\hline AST-487 & Erk7 & 94 & PKC-412 & DYRK1B & 330 \\
\hline AST-487 & FER & 590 & PKC-412 & EGFR & 1300 \\
\hline AST-487 & FES & 360 & PKC-412 & ErbB4 & 3100 \\
\hline AST-487 & FGFR1 & 620 & PKC-412 & FER & 1200 \\
\hline AST-487 & FGFR2 & 1900 & PKC-412 & FGFR1 & 1600 \\
\hline AST-487 & FGFR4 & 1400 & PKC-412 & FGFR2 & 2400 \\
\hline AST-487 & FGR & 190 & PKC-412 & FGFR3 & 1700 \\
\hline AST-487 & FLT1 & 86 & PKC-412 & FGR & 730 \\
\hline AST-487 & FLT3 & 0.79 & PKC-412 & FLT1 & 450 \\
\hline AST-487 & FLT4 & 120 & PKC-412 & FLT3 & 11 \\
\hline AST-487 & FMS & 5.8 & PKC-412 & FLT4 & 670 \\
\hline AST-487 & FRK & 26 & PKC-412 & FMS & 330 \\
\hline AST-487 & Fused & 1300 & PKC-412 & FRK & 2400 \\
\hline AST-487 & FYN & 50 & PKC-412 & FYN & 2100 \\
\hline AST-487 & GAK & 1300 & PKC-412 & GAK & 380 \\
\hline AST-487 & $\mathrm{HCK}$ & 880 & PKC-412 & GCN2 & 39 \\
\hline AST-487 & HGK & 15 & PKC-412 & GSK3A & 2500 \\
\hline AST-487 & $\mathrm{HH} 498$ & 170 & PKC-412 & GSK3B & 1800 \\
\hline AST-487 & HPK1 & 210 & PKC-412 & $\mathrm{HCK}$ & 720 \\
\hline AST-487 & IRR & 1400 & PKC-412 & HGK & 650 \\
\hline AST-487 & ITK & 990 & PKC-412 & HPK1 & 2100 \\
\hline AST-487 & JAK2 & 910 & PKC-412 & $\mathrm{IKKe}$ & 160 \\
\hline AST-487 & JAK3 & 260 & PKC-412 & IRAK3 & 180 \\
\hline AST-487 & JNK1 & 460 & PKC-412 & JAK2 & 94 \\
\hline AST-487 & JNK2 & 56 & PKC-412 & JAK3 & 12 \\
\hline AST-487 & JNK3 & 760 & PKC-412 & JNK1 & 4400 \\
\hline AST-487 & KDR & 200 & PKC-412 & JNK3 & 3600 \\
\hline AST-487 & KHS1 & 350 & PKC-412 & KDR & 3200 \\
\hline AST-487 & KHS2 & 190 & PKC-412 & KHS1 & 1100 \\
\hline AST-487 & $\mathrm{KIT}$ & 5.4 & PKC-412 & KHS2 & 120 \\
\hline AST-487 & LATS1 & 470 & PKC-412 & KIT & 220 \\
\hline AST-487 & LATS2 & 3000 & PKC-412 & LATS1 & 1100 \\
\hline AST-487 & LCK & 11 & PKC-412 & LATS2 & 2200 \\
\hline AST-487 & LOK & 0.92 & PKC-412 & LCK & 280 \\
\hline AST-487 & LTK & 160 & PKC-412 & LKB1 & 350 \\
\hline AST-487 & LYN & 14 & PKC-412 & LOK & 590 \\
\hline AST-487 & MELK & 830 & PKC-412 & LTK & 3000 \\
\hline AST-487 & MER & 370 & PKC-412 & LYN & 4200 \\
\hline AST-487 & MET & 4400 & PKC-412 & MAP2K3 & 5300 \\
\hline AST-487 & MNK1 & 26 & PKC-412 & MAP2K4 & 4800 \\
\hline
\end{tabular}




\begin{tabular}{|c|c|c|c|c|c|}
\hline AST-487 & MNK2 & 3 & PKC-412 & MARK1 & 170 \\
\hline AST-487 & MST2 & 860 & PKC-412 & MARK2 & 100 \\
\hline AST-487 & MUSK & 3.1 & PKC-412 & MARK3 & 21 \\
\hline AST-487 & MYO3A & 41 & PKC-412 & MARK4 & 370 \\
\hline AST-487 & MYO3B & 84 & PKC-412 & MELK & 280 \\
\hline AST-487 & NEK5 & 1100 & PKC-412 & MER & 2900 \\
\hline AST-487 & NLK & 160 & PKC-412 & MET & 690 \\
\hline AST-487 & p38a & 73 & PKC-412 & MLK1 & 15 \\
\hline AST-487 & p38b & 430 & PKC-412 & MLK2 & 790 \\
\hline AST-487 & p38g & 29 & PKC-412 & MLK3 & 17 \\
\hline AST-487 & PCTAIRE1 & 420 & PKC-412 & MNK2 & 950 \\
\hline AST-487 & PCTAIRE2 & 15 & PKC-412 & MPSK1 & 280 \\
\hline AST-487 & PCTAIRE3 & 54 & PKC-412 & MST1 & 40 \\
\hline AST-487 & PDGFRa & 27 & PKC-412 & MST2 & 220 \\
\hline AST-487 & PDGFRb & 8.1 & PKC-412 & NuaK1 & 41 \\
\hline AST-487 & PFTAIRE1 & 17 & PKC-412 & PAK1 & 2100 \\
\hline AST-487 & PITSLRE & 1400 & PKC-412 & PAK2 & 3300 \\
\hline AST-487 & PITSLRE2 & 1100 & PKC-412 & PAK3 & 180 \\
\hline AST-487 & PKACa & 3700 & PKC-412 & PDGFRa & 380 \\
\hline AST-487 & PKACb & 260 & PKC-412 & PDGFRb & 110 \\
\hline AST-487 & PKCt & 2200 & PKC-412 & PDK1 & 190 \\
\hline AST-487 & PKD1 & 1200 & PKC-412 & PHKg1 & 900 \\
\hline AST-487 & PKD2 & 950 & PKC-412 & PHKg2 & 1400 \\
\hline AST-487 & PKD3 & 1500 & PKC-412 & PIM1 & 560 \\
\hline AST-487 & PKR & 1100 & PKC-412 & PIM3 & 560 \\
\hline AST-487 & PYK2 & 520 & PKC-412 & PIP5K1A & 310 \\
\hline AST-487 & RAF1 & 930 & PKC-412 & PIP5K2B & 270 \\
\hline AST-487 & RET & 17 & PKC-412 & PKACa & 720 \\
\hline AST-487 & RIPK1 & 210 & PKC-412 & PKACb & 240 \\
\hline AST-487 & RIPK2 & 1100 & PKC-412 & PKCd & 320 \\
\hline AST-487 & ROS & 1700 & PKC-412 & PKCe & 540 \\
\hline AST-487 & SgK288 & 630 & PKC-412 & PKCh & 290 \\
\hline AST-487 & SIK & 1700 & PKC-412 & PKCt & 920 \\
\hline AST-487 & skMLCK & 330 & PKC-412 & PKD2 & 6300 \\
\hline AST-487 & SLK & 23 & PKC-412 & PKD3 & 3200 \\
\hline AST-487 & SRC & 340 & PKC-412 & PKG1 & 250 \\
\hline AST-487 & SRM & 72 & PKC-412 & PKG2 & 74 \\
\hline AST-487 & STK33 & 1200 & PKC-412 & PKN1 & 9.3 \\
\hline AST-487 & SYK & 600 & PKC-412 & PKN2 & 15 \\
\hline AST-487 & TESK1 & 2400 & PKC-412 & PKR & 6900 \\
\hline AST-487 & TGFbR2 & 260 & PKC-412 & PLK4 & 66 \\
\hline AST-487 & TIE1 & 0.29 & PKC-412 & PRKX & 960 \\
\hline AST-487 & TIE2 & 19 & PKC-412 & PYK2 & 660 \\
\hline AST-487 & TNIK & 69 & PKC-412 & QIK & 560 \\
\hline
\end{tabular}




\begin{tabular}{|c|c|c|c|c|c|}
\hline AST-487 & TNK1 & 60 & PKC-412 & RET & 350 \\
\hline AST-487 & TRKA & 320 & PKC-412 & RIOK1 & 1200 \\
\hline AST-487 & TRKB & 190 & PKC-412 & RIOK3 & 420 \\
\hline AST-487 & TRKC & 34 & PKC-412 & ROS & 430 \\
\hline AST-487 & TTK & 230 & PKC-412 & SgK085 & 640 \\
\hline AST-487 & TYK2 & 2100 & PKC-412 & SIK & 160 \\
\hline AST-487 & YES & 260 & PKC-412 & SLK & 220 \\
\hline AST-487 & YSK1 & 1200 & PKC-412 & smMLCK & 2000 \\
\hline AST-487 & ZAK & 2.3 & PKC-412 & SNRK & 63 \\
\hline AZD-1152HQPA & AurA & 590 & PKC-412 & SRC & 1200 \\
\hline AZD-1152HQPA & AurB & 7.2 & PKC-412 & SRPK1 & 42 \\
\hline AZD-1152HQPA & AurC & 4.4 & PKC-412 & SRPK2 & 330 \\
\hline AZD-1152HQPA & $A X L$ & 390 & PKC-412 & SYK & 88 \\
\hline AZD-1152HQPA & BLK & 1500 & PKC-412 & TIE1 & 1400 \\
\hline AZD-1152HQPA & CDK7 & 6200 & PKC-412 & TIE2 & 1900 \\
\hline AZD-1152HQPA & CRIK & 5700 & PKC-412 & TNIK & 1600 \\
\hline AZD-1152HQPA & DDR1 & 1800 & PKC-412 & TNK1 & 83 \\
\hline AZD-1152HQPA & EGFR & 450 & PKC-412 & TRKA & 380 \\
\hline AZD-1152HQPA & ErbB2 & 4300 & PKC-412 & TRKB & 310 \\
\hline AZD-1152HQPA & ErbB4 & 470 & PKC-412 & TRKC & 1700 \\
\hline AZD-1152HQPA & FLT1 & 110 & PKC-412 & TSSK1 & 4800 \\
\hline AZD-1152HQPA & FLT3 & 8.2 & PKC-412 & TTK & 350 \\
\hline AZD-1152HQPA & FMS & 1400 & PKC-412 & TYK2 & 250 \\
\hline AZD-1152HQPA & Fused & 4600 & PKC-412 & YES & 950 \\
\hline AZD-1152HQPA & HCK & 3900 & PKC-412 & YSK1 & 1800 \\
\hline AZD-1152HQPA & KDR & 500 & PKC-412 & ZAP70 & 2600 \\
\hline AZD-1152HQPA & KHS1 & 160 & PTK-787 & BRK & 2400 \\
\hline AZD-1152HQPA & $\mathrm{KIT}$ & 17 & PTK-787 & CRIK & 8800 \\
\hline AZD-1152HQPA & LCK & 380 & PTK-787 & DDR1 & 270 \\
\hline AZD-1152HQPA & LOK & 2800 & PTK-787 & FLT1 & 9.6 \\
\hline AZD-1152HQPA & LTK & 3200 & PTK-787 & FLT4 & 330 \\
\hline AZD-1152HQPA & LYN & 1600 & PTK-787 & FMS & 18 \\
\hline AZD-1152HQPA & MAP2K2 & 5700 & PTK-787 & FRK & 1800 \\
\hline AZD-1152HQPA & MUSK & 1500 & PTK-787 & KDR & 62 \\
\hline AZD-1152HQPA & PCTAIRE1 & 6300 & PTK-787 & KIT & 5.1 \\
\hline AZD-1152HQPA & PDGFRa & 38 & PTK-787 & PDGFRa & 96 \\
\hline AZD-1152HQPA & PDGFRb & 41 & PTK-787 & PDGFRb & 25 \\
\hline AZD-1152HQPA & PITSLRE & 1200 & PTK-787 & ZAK & 4400 \\
\hline AZD-1152HQPA & PITSLRE2 & 1000 & Roscovitine/CYC-202 & ALK & 2300 \\
\hline AZD-1152HQPA & PLK4 & 4700 & Roscovitine/CYC-202 & CDK2 & 3400 \\
\hline AZD-1152HQPA & RET & 80 & Roscovitine/CYC-202 & CDK5 & 1900 \\
\hline AZD-1152HQPA & RIPK2 & 1800 & Roscovitine/CYC-202 & CDK7 & 1800 \\
\hline AZD-1152HQPA & SgK288 & 1500 & Roscovitine/CYC-202 & CK1d & 260 \\
\hline AZD-1152HQPA & SRM & 7900 & Roscovitine/CYC-202 & CK1e & 320 \\
\hline
\end{tabular}




\begin{tabular}{|c|c|c|c|c|c|}
\hline AZD-1152HQPA & TIE1 & 350 & Roscovitine/CYC-202 & CK1g3 & 2900 \\
\hline AZD-1152HQPA & TIE2 & 1900 & Roscovitine/CYC-202 & CLK1 & 1200 \\
\hline AZD-1152HQPA & TTK & 8500 & Roscovitine/CYC-202 & CLK2 & 700 \\
\hline AZD-1152HQPA & YES & 3800 & Roscovitine/CYC-202 & DYRK1B & 1100 \\
\hline AZD-1152HQPA & ZAK & 1700 & Roscovitine/CYC-202 & TTK & 1600 \\
\hline BIRB-796 & ABL1 & 3400 & SB-202190 & ACTR2B & 4800 \\
\hline BIRB-796 & BLK & 680 & SB-202190 & ADCK4 & 4900 \\
\hline BIRB-796 & BRAF & 2700 & SB-202190 & ALK4 & 950 \\
\hline BIRB-796 & CDK11 & 200 & SB-202190 & BRAF & 4800 \\
\hline BIRB-796 & CDK5 & 2000 & SB-202190 & BRK & 6800 \\
\hline BIRB-796 & CDK8 & 220 & SB-202190 & CK1a & 1900 \\
\hline BIRB-796 & CRIK & 2100 & SB-202190 & CK1d & 59 \\
\hline BIRB-796 & DDR1 & 1.9 & SB-202190 & CK1e & 170 \\
\hline BIRB-796 & DDR2 & 33 & SB-202190 & CRIK & 510 \\
\hline BIRB-796 & DMPK2 & 1200 & SB-202190 & DDR1 & 1100 \\
\hline BIRB-796 & EGFR & 7000 & SB-202190 & DDR2 & 1600 \\
\hline BIRB-796 & EphA1 & 9800 & SB-202190 & DMPK2 & 640 \\
\hline BIRB-796 & EphA2 & 6200 & SB-202190 & EGFR & 2600 \\
\hline BIRB-796 & EphA3 & 880 & SB-202190 & EphA6 & 2200 \\
\hline BIRB-796 & EphA4 & 2900 & SB-202190 & ErbB4 & 4900 \\
\hline BIRB-796 & EphA5 & 1400 & SB-202190 & FRK & 3100 \\
\hline BIRB-796 & EphA6 & 2000 & SB-202190 & Fused & 790 \\
\hline BIRB-796 & EphA7 & 860 & SB-202190 & GAK & 53 \\
\hline BIRB-796 & EphA8 & 270 & SB-202190 & GSK3B & 2700 \\
\hline BIRB-796 & EphB1 & 4900 & SB-202190 & JNK1 & 2400 \\
\hline BIRB-796 & EphB2 & 440 & SB-202190 & JNK2 & 210 \\
\hline BIRB-796 & Erk7 & 3000 & SB-202190 & JNK3 & 42 \\
\hline BIRB-796 & FAK & 7100 & SB-202190 & LATS2 & 1700 \\
\hline BIRB-796 & FGFR1 & 4300 & SB-202190 & MAP2K2 & 9600 \\
\hline BIRB-796 & FLT1 & 410 & SB-202190 & MRCKb & 4200 \\
\hline BIRB-796 & FLT3 & 2300 & SB-202190 & NLK & 28 \\
\hline BIRB-796 & FLT4 & 7100 & SB-202190 & p38a & 9.8 \\
\hline BIRB-796 & FMS & 8100 & SB-202190 & p38b & 32 \\
\hline BIRB-796 & FRK & 1200 & SB-202190 & p38g & 3300 \\
\hline BIRB-796 & HGK & 90 & SB-202190 & PKACa & 1700 \\
\hline BIRB-796 & JNK2 & 7.3 & SB-202190 & PKACb & 530 \\
\hline BIRB-796 & JNK3 & 110 & SB-202190 & RAF1 & 1900 \\
\hline BIRB-796 & KDR & 3900 & SB-202190 & RIPK2 & 150 \\
\hline BIRB-796 & KIT & 170 & SB-202190 & SLK & 3200 \\
\hline BIRB-796 & LCK & 1200 & SB-202190 & TNIK & 1600 \\
\hline BIRB-796 & LOK & 12 & SB-202190 & TTK & 4500 \\
\hline BIRB-796 & LTK & 1400 & SB-202190 & YANK2 & 4900 \\
\hline BIRB-796 & LYN & 2700 & SB-203580 & ADCK4 & 3100 \\
\hline BIRB-796 & MER & 3700 & SB-203580 & ALK4 & 3000 \\
\hline
\end{tabular}




\begin{tabular}{|c|c|c|c|c|c|}
\hline BIRB-796 & MNK2 & 1600 & SB-203580 & BRAF & 710 \\
\hline BIRB-796 & MRCKa & 9400 & SB-203580 & BRK & 3900 \\
\hline BIRB-796 & MRCKb & 910 & SB-203580 & CK1a & 1700 \\
\hline BIRB-796 & MUSK & 1900 & SB-203580 & CK1d & 37 \\
\hline BIRB-796 & NLK & 1000 & SB-203580 & CK1e & 100 \\
\hline BIRB-796 & p38a & 0.37 & SB-203580 & CRIK & 420 \\
\hline BIRB-796 & p38b & 1500 & SB-203580 & DDR1 & 1000 \\
\hline BIRB-796 & p38g & 19 & SB-203580 & DDR2 & 5000 \\
\hline BIRB-796 & PDGFRa & 1200 & SB-203580 & DMPK2 & 400 \\
\hline BIRB-796 & PDGFRb & 1100 & SB-203580 & EGFR & 1700 \\
\hline BIRB-796 & PYK2 & 990 & SB-203580 & EphA6 & 1200 \\
\hline BIRB-796 & RET & 500 & SB-203580 & FRK & 1800 \\
\hline BIRB-796 & SgK288 & 1000 & SB-203580 & Fused & 1300 \\
\hline BIRB-796 & SLK & 150 & SB-203580 & GAK & 19 \\
\hline BIRB-796 & TIE1 & 8.3 & SB-203580 & GSK3B & 1700 \\
\hline BIRB-796 & TIE2 & 20 & SB-203580 & HGK & 3700 \\
\hline BIRB-796 & TNIK & 140 & SB-203580 & $\mathrm{HH} 498$ & 3500 \\
\hline BIRB-796 & TRKA & 1000 & SB-203580 & JNK1 & 1100 \\
\hline BIRB-796 & TRKB & 570 & SB-203580 & JNK2 & 130 \\
\hline BIRB-796 & TRKC & 190 & SB-203580 & JNK3 & 35 \\
\hline BIRB-796 & TTK & 3300 & SB-203580 & LCK & 2800 \\
\hline BIRB-796 & YES & 4900 & SB-203580 & MAP2K2 & 9600 \\
\hline BIRB-796 & ZAK & 860 & SB-203580 & MRCKa & 6200 \\
\hline BMS-387032/SNS-032 & AurA & 7400 & SB-203580 & MRCKb & 2700 \\
\hline BMS-387032/SNS-032 & CaMLCK & 1700 & SB-203580 & NLK & 25 \\
\hline BMS-387032/SNS-032 & CDK11 & 950 & SB-203580 & p38a & 12 \\
\hline BMS-387032/SNS-032 & CDK2 & 69 & SB-203580 & p38b & 70 \\
\hline BMS-387032/SNS-032 & CDK3 & 56 & SB-203580 & p38g & 1500 \\
\hline BMS-387032/SNS-032 & CDK5 & 740 & SB-203580 & RAF1 & 980 \\
\hline BMS-387032/SNS-032 & CDK7 & 31 & SB-203580 & RIPK2 & 24 \\
\hline BMS-387032/SNS-032 & CDK8 & 1200 & SB-203580 & SLK & 3700 \\
\hline BMS-387032/SNS-032 & CDK9 & 76 & SB-203580 & SRC & 5300 \\
\hline BMS-387032/SNS-032 & CK1d & 1800 & SB-203580 & TGFbR1 & 7100 \\
\hline BMS-387032/SNS-032 & CK1e & 950 & SB-203580 & TGFbR2 & 1800 \\
\hline BMS-387032/SNS-032 & CLK1 & 410 & SB-203580 & TNIK & 820 \\
\hline BMS-387032/SNS-032 & CLK2 & 1100 & SB-203580 & TTK & 2500 \\
\hline BMS-387032/SNS-032 & CLK4 & 800 & SB-203580 & TXK & 4500 \\
\hline BMS-387032/SNS-032 & DCAMKL1 & 1600 & SB-203580 & YANK2 & 2100 \\
\hline BMS-387032/SNS-032 & DCAMKL2 & 1000 & SB-203580 & ZAK & 4400 \\
\hline BMS-387032/SNS-032 & DCAMKL3 & 1600 & SB-431542 & ALK4 & 190 \\
\hline BMS-387032/SNS-032 & DRAK1 & 440 & SB-431542 & CK1a & 5900 \\
\hline BMS-387032/SNS-032 & DYRK1B & 200 & SB-431542 & CK1d & 170 \\
\hline BMS-387032/SNS-032 & ErK3 & 500 & SB-431542 & CK1e & 260 \\
\hline BMS-387032/SNS-032 & ErK4 & 4100 & SB-431542 & RIPK2 & 3400 \\
\hline
\end{tabular}




\begin{tabular}{|c|c|c|c|c|c|}
\hline BMS-387032/SNS-032 & ErK5 & 650 & SB-431542 & TGFbR1 & 170 \\
\hline BMS-387032/SNS-032 & Erk7 & 120 & Sorafenib & ABL1 & 680 \\
\hline BMS-387032/SNS-032 & Fused & 260 & Sorafenib & ABL2 & 2900 \\
\hline BMS-387032/SNS-032 & GAK & 1800 & Sorafenib & AurB & 440 \\
\hline BMS-387032/SNS-032 & GSK3A & 28 & Sorafenib & AurC & 210 \\
\hline BMS-387032/SNS-032 & GSK3B & 37 & Sorafenib & $A X L$ & 4500 \\
\hline BMS-387032/SNS-032 & IRAK3 & 2900 & Sorafenib & BRAF & 540 \\
\hline BMS-387032/SNS-032 & MELK & 5600 & Sorafenib & CDK11 & 250 \\
\hline BMS-387032/SNS-032 & MNK1 & 3800 & Sorafenib & CDK7 & 140 \\
\hline BMS-387032/SNS-032 & PAK3 & 3500 & Sorafenib & CDK8 & 310 \\
\hline BMS-387032/SNS-032 & PCTAIRE1 & 7.1 & Sorafenib & CRIK & 6200 \\
\hline BMS-387032/SNS-032 & PCTAIRE2 & 13 & Sorafenib & DDR1 & 1.5 \\
\hline BMS-387032/SNS-032 & PCTAIRE3 & 44 & Sorafenib & DDR2 & 6.6 \\
\hline BMS-387032/SNS-032 & PFTAIRE1 & 690 & Sorafenib & EphA1 & 3100 \\
\hline BMS-387032/SNS-032 & PHKg1 & 3800 & Sorafenib & EphA2 & 2000 \\
\hline BMS-387032/SNS-032 & PITSLRE & 98 & Sorafenib & EphA3 & 1900 \\
\hline BMS-387032/SNS-032 & PITSLRE2 & 48 & Sorafenib & EphA4 & 3000 \\
\hline BMS-387032/SNS-032 & PKD1 & 1200 & Sorafenib & EphA6 & 370 \\
\hline BMS-387032/SNS-032 & PKD2 & 3900 & Sorafenib & EphA7 & 5300 \\
\hline BMS-387032/SNS-032 & PKD3 & 750 & Sorafenib & EphA8 & 2400 \\
\hline BMS-387032/SNS-032 & skMLCK & 980 & Sorafenib & EphB1 & 3000 \\
\hline BMS-387032/SNS-032 & SNRK & 1700 & Sorafenib & EphB2 & 1900 \\
\hline BMS-387032/SNS-032 & TGFbR2 & 2400 & Sorafenib & EphB4 & 1800 \\
\hline CHIR-258/TKI-258 & AAK1 & 170 & Sorafenib & Erk7 & 46 \\
\hline CHIR-258/TKI-258 & ABL1 & 870 & Sorafenib & FGFR1 & 2800 \\
\hline CHIR-258/TKI-258 & ALK & 3500 & Sorafenib & FGFR2 & 2700 \\
\hline CHIR-258/TKI-258 & AMPKa1 & 520 & Sorafenib & FLT1 & 31 \\
\hline CHIR-258/TKI-258 & AMPKa2 & 820 & Sorafenib & FLT3 & 13 \\
\hline CHIR-258/TKI-258 & AurB & 280 & Sorafenib & FLT4 & 95 \\
\hline CHIR-258/TKI-258 & AurC & 1100 & Sorafenib & FMS & 28 \\
\hline CHIR-258/TKI-258 & AXL & 3800 & Sorafenib & FRK & 510 \\
\hline CHIR-258/TKI-258 & BIKE & 54 & Sorafenib & Fused & 3800 \\
\hline CHIR-258/TKI-258 & BLK & 190 & Sorafenib & HGK & 4800 \\
\hline CHIR-258/TKI-258 & BMPR2 & 3100 & Sorafenib & $\mathrm{HH} 498$ & 280 \\
\hline CHIR-258/TKI-258 & CaMKK1 & 3100 & Sorafenib & $\mathrm{KDR}$ & 59 \\
\hline CHIR-258/TKI-258 & CaMLCK & 2 & Sorafenib & KHS1 & 1600 \\
\hline CHIR-258/TKI-258 & CDK7 & 2600 & Sorafenib & $\mathrm{KIT}$ & 31 \\
\hline CHIR-258/TKI-258 & CHK1 & 1200 & Sorafenib & LCK & 2700 \\
\hline CHIR-258/TKI-258 & CK2a1 & 1400 & Sorafenib & LIMK1 & 1600 \\
\hline CHIR-258/TKI-258 & CK2a2 & 1600 & Sorafenib & LOK & 150 \\
\hline CHIR-258/TKI-258 & CLK1 & 6800 & Sorafenib & LYN & 3000 \\
\hline CHIR-258/TKI-258 & CLK2 & 1700 & Sorafenib & MER & 3600 \\
\hline CHIR-258/TKI-258 & CLK4 & 6500 & Sorafenib & MNK1 & 230 \\
\hline CHIR-258/TKI-258 & CRIK & 8200 & Sorafenib & MNK2 & 130 \\
\hline
\end{tabular}




\begin{tabular}{|c|c|c|c|c|c|}
\hline CHIR-258/TKI-258 & DCAMKL3 & 1300 & Sorafenib & MUSK & 130 \\
\hline CHIR-258/TKI-258 & DDR1 & 2500 & Sorafenib & NLK & 640 \\
\hline CHIR-258/TKI-258 & DLK & 2100 & Sorafenib & p38a & 370 \\
\hline CHIR-258/TKI-258 & DRAK1 & 830 & Sorafenib & p38b & 230 \\
\hline CHIR-258/TKI-258 & DRAK2 & 1800 & Sorafenib & p38g & 7600 \\
\hline CHIR-258/TKI-258 & FGFR1 & 150 & Sorafenib & PCTAIRE2 & 1600 \\
\hline CHIR-258/TKI-258 & FGFR2 & 410 & Sorafenib & PDGFRa & 62 \\
\hline CHIR-258/TKI-258 & FGFR3 & 230 & Sorafenib & PDGFRb & 37 \\
\hline CHIR-258/TKI-258 & FGFR4 & 4300 & Sorafenib & PFTAIRE1 & 2900 \\
\hline CHIR-258/TKI-258 & FGR & 190 & Sorafenib & PLK4 & 4500 \\
\hline CHIR-258/TKI-258 & FLT1 & 69 & Sorafenib & RAF1 & 230 \\
\hline CHIR-258/TKI-258 & FLT3 & 0.64 & Sorafenib & RET & 13 \\
\hline CHIR-258/TKI-258 & FLT4 & 580 & Sorafenib & RIPK2 & 1300 \\
\hline CHIR-258/TKI-258 & FMS & 60 & Sorafenib & SLK & 1000 \\
\hline CHIR-258/TKI-258 & FRK & 2300 & Sorafenib & SRM & 9800 \\
\hline CHIR-258/TKI-258 & FYN & 440 & Sorafenib & STK33 & 2400 \\
\hline CHIR-258/TKI-258 & GAK & 490 & Sorafenib & TIE1 & 68 \\
\hline CHIR-258/TKI-258 & GCN2 & 250 & Sorafenib & TIE2 & 2100 \\
\hline CHIR-258/TKI-258 & GSK3B & 350 & Sorafenib & TNK1 & 2300 \\
\hline CHIR-258/TKI-258 & $\mathrm{HCK}$ & 3300 & Sorafenib & TRKA & 6300 \\
\hline CHIR-258/TKI-258 & HGK & 160 & Sorafenib & TRKB & 2100 \\
\hline CHIR-258/TKI-258 & HPK1 & 44 & Sorafenib & TRKC & 600 \\
\hline CHIR-258/TKI-258 & IKKe & 480 & Sorafenib & TTK & 3500 \\
\hline CHIR-258/TKI-258 & ITK & 530 & Sorafenib & ZAK & 6.3 \\
\hline CHIR-258/TKI-258 & JAK2 & 7000 & Staurosporine & AAK1 & 1.2 \\
\hline CHIR-258/TKI-258 & JAK3 & 8700 & Staurosporine & ABL1 & 290 \\
\hline CHIR-258/TKI-258 & KDR & 68 & Staurosporine & ABL2 & 110 \\
\hline CHIR-258/TKI-258 & KHS1 & 620 & Staurosporine & ACK & 16 \\
\hline CHIR-258/TKI-258 & KHS2 & 560 & Staurosporine & ACTR2B & 4600 \\
\hline CHIR-258/TKI-258 & KIT & 7.5 & Staurosporine & AKT1 & 20 \\
\hline CHIR-258/TKI-258 & LATS1 & 1700 & Staurosporine & AKT2 & 44 \\
\hline CHIR-258/TKI-258 & LATS2 & 530 & Staurosporine & AKT3 & 170 \\
\hline CHIR-258/TKI-258 & LCK & 250 & Staurosporine & ALK & 32 \\
\hline CHIR-258/TKI-258 & LKB1 & 3700 & Staurosporine & ALK2 & 470 \\
\hline CHIR-258/TKI-258 & LOK & 550 & Staurosporine & ALK4 & 680 \\
\hline CHIR-258/TKI-258 & LYN & 750 & Staurosporine & AMPKa1 & 3.7 \\
\hline CHIR-258/TKI-258 & MAP2K1 & 520 & Staurosporine & AMPKa2 & 12 \\
\hline CHIR-258/TKI-258 & MAP2K2 & 350 & Staurosporine & AurA & 110 \\
\hline CHIR-258/TKI-258 & MAP3K4 & 5500 & Staurosporine & AurB & 19 \\
\hline CHIR-258/TKI-258 & MARK1 & 2300 & Staurosporine & AurC & 23 \\
\hline CHIR-258/TKI-258 & MARK2 & 1500 & Staurosporine & $A X L$ & 6.8 \\
\hline CHIR-258/TKI-258 & MARK3 & 3400 & Staurosporine & BIKE & 5.6 \\
\hline CHIR-258/TKI-258 & MARK4 & 1700 & Staurosporine & BLK & 15 \\
\hline CHIR-258/TKI-258 & MELK & 1600 & Staurosporine & BMPR1A & 3700 \\
\hline
\end{tabular}




\begin{tabular}{|c|c|c|c|c|c|}
\hline CHIR-258/TKI-258 & MER & 1800 & Staurosporine & BMX & 170 \\
\hline CHIR-258/TKI-258 & MLK1 & 9900 & Staurosporine & BRK & 2300 \\
\hline CHIR-258/TKI-258 & MLK3 & 2200 & Staurosporine & BRSK1 & 26 \\
\hline CHIR-258/TKI-258 & MST1 & 200 & Staurosporine & BRSK2 & 3.5 \\
\hline CHIR-258/TKI-258 & MST2 & 480 & Staurosporine & BTK & 210 \\
\hline CHIR-258/TKI-258 & MST3 & 430 & Staurosporine & CaMK1a & 27 \\
\hline CHIR-258/TKI-258 & MST4 & 540 & Staurosporine & CaMK1d & 1.1 \\
\hline CHIR-258/TKI-258 & MUSK & 830 & Staurosporine & CaMK1g & 23 \\
\hline CHIR-258/TKI-258 & NEK2 & 2000 & Staurosporine & CaMK2a & 0.16 \\
\hline CHIR-258/TKI-258 & NEK5 & 2300 & Staurosporine & CaMK2b & 1.3 \\
\hline CHIR-258/TKI-258 & NEK6 & 1500 & Staurosporine & CaMK2d & 0.32 \\
\hline CHIR-258/TKI-258 & NEK7 & 5600 & Staurosporine & CaMK2g & 0.55 \\
\hline CHIR-258/TKI-258 & NEK9 & 9100 & Staurosporine & CaMK4 & 41 \\
\hline CHIR-258/TKI-258 & NuaK1 & 240 & Staurosporine & CaMKK1 & 0.039 \\
\hline CHIR-258/TKI-258 & PAK3 & 770 & Staurosporine & CaMKK2 & 0.16 \\
\hline CHIR-258/TKI-258 & PCTAIRE1 & 430 & Staurosporine & CaMLCK & 140 \\
\hline CHIR-258/TKI-258 & PCTAIRE2 & 3000 & Staurosporine & CDK11 & 190 \\
\hline CHIR-258/TKI-258 & PDGFRa & 54 & Staurosporine & CDK2 & 7 \\
\hline CHIR-258/TKI-258 & PDGFRb & 3.8 & Staurosporine & CDK3 & 30 \\
\hline CHIR-258/TKI-258 & PHKg1 & 640 & Staurosporine & CDK5 & 84 \\
\hline CHIR-258/TKI-258 & PHKg2 & 9000 & Staurosporine & CDK7 & 45 \\
\hline CHIR-258/TKI-258 & PIP5K1A & 1900 & Staurosporine & CDK8 & 510 \\
\hline CHIR-258/TKI-258 & PIP5K2B & 260 & Staurosporine & CDK9 & 100 \\
\hline CHIR-258/TKI-258 & $\mathrm{PKCt}$ & 3200 & Staurosporine & $\mathrm{CHK} 1$ & 3.2 \\
\hline CHIR-258/TKI-258 & PKN1 & 180 & Staurosporine & CK1a & 250 \\
\hline CHIR-258/TKI-258 & PKN2 & 9900 & Staurosporine & CK1d & 2400 \\
\hline CHIR-258/TKI-258 & PKR & 2800 & Staurosporine & CK1e & 73 \\
\hline CHIR-258/TKI-258 & QIK & 3700 & Staurosporine & CK1g2 & 780 \\
\hline CHIR-258/TKI-258 & RET & 71 & Staurosporine & CK2a1 & 36 \\
\hline CHIR-258/TKI-258 & RIOK1 & 420 & Staurosporine & CK2a2 & 6.5 \\
\hline CHIR-258/TKI-258 & RIOK3 & 690 & Staurosporine & CLK1 & 32 \\
\hline CHIR-258/TKI-258 & RIPK1 & 320 & Staurosporine & CLK2 & 8.2 \\
\hline CHIR-258/TKI-258 & SgK085 & 80 & Staurosporine & CLK3 & 910 \\
\hline CHIR-258/TKI-258 & SgK288 & 79 & Staurosporine & CLK4 & 9.6 \\
\hline CHIR-258/TKI-258 & skMLCK & 92 & Staurosporine & CRIK & 340 \\
\hline CHIR-258/TKI-258 & SLK & 300 & Staurosporine & CSK & 330 \\
\hline CHIR-258/TKI-258 & smMLCK & 3500 & Staurosporine & DAPK1 & 1.4 \\
\hline CHIR-258/TKI-258 & SNRK & 130 & Staurosporine & DAPK2 & 1.6 \\
\hline CHIR-258/TKI-258 & $\mathrm{SRC}$ & 1800 & Staurosporine & DAPK3 & 1 \\
\hline CHIR-258/TKI-258 & SRPK1 & 420 & Staurosporine & DCAMKL1 & 110 \\
\hline CHIR-258/TKI-258 & SRPK2 & 290 & Staurosporine & DCAMKL2 & 73 \\
\hline CHIR-258/TKI-258 & TIE1 & 4000 & Staurosporine & DCAMKL3 & 17 \\
\hline CHIR-258/TKI-258 & TLK2 & 2600 & Staurosporine & DDR1 & 19 \\
\hline CHIR-258/TKI-258 & TNIK & 24 & Staurosporine & DDR2 & 42 \\
\hline
\end{tabular}




\begin{tabular}{|c|c|c|c|c|c|}
\hline CHIR-258/TKI-258 & TRKA & 100 & Staurosporine & DLK & 1500 \\
\hline CHIR-258/TKI-258 & TRKB & 720 & Staurosporine & DMPK1 & 3.5 \\
\hline CHIR-258/TKI-258 & TRKC & 190 & Staurosporine & DMPK2 & 37 \\
\hline CHIR-258/TKI-258 & TYRO3 & 9300 & Staurosporine & DRAK1 & 14 \\
\hline CHIR-258/TKI-258 & Wee1 & 1200 & Staurosporine & DRAK2 & 21 \\
\hline CHIR-258/TKI-258 & YES & 580 & Staurosporine & DYRK1B & 28 \\
\hline CHIR-258/TKI-258 & YSK1 & 1200 & Staurosporine & EGFR & 370 \\
\hline CHIR-265/RAF-265 & ABL1 & 310 & Staurosporine & EphA1 & 300 \\
\hline CHIR-265/RAF-265 & ABL2 & 1900 & Staurosporine & EphA2 & 1200 \\
\hline CHIR-265/RAF-265 & AMPKa1 & 4500 & Staurosporine & EphA3 & 27 \\
\hline CHIR-265/RAF-265 & BRAF & 1200 & Staurosporine & EphA4 & 290 \\
\hline CHIR-265/RAF-265 & BRK & 3600 & Staurosporine & EphA5 & 95 \\
\hline CHIR-265/RAF-265 & CDK11 & 5100 & Staurosporine & EphA6 & 290 \\
\hline CHIR-265/RAF-265 & CRIK & 87 & Staurosporine & EphA7 & 630 \\
\hline CHIR-265/RAF-265 & DDR1 & 13 & Staurosporine & EphA8 & 190 \\
\hline CHIR-265/RAF-265 & DDR2 & 960 & Staurosporine & EphB1 & 240 \\
\hline CHIR-265/RAF-265 & EphA2 & 2900 & Staurosporine & EphB2 & 2100 \\
\hline CHIR-265/RAF-265 & EphA6 & 1500 & Staurosporine & ErbB4 & 770 \\
\hline CHIR-265/RAF-265 & EphA8 & 1100 & Staurosporine & ErK1 & 8400 \\
\hline CHIR-265/RAF-265 & FGFR1 & 4700 & Staurosporine & ErK2 & 7300 \\
\hline CHIR-265/RAF-265 & FLT1 & 800 & Staurosporine & ErK5 & 1600 \\
\hline CHIR-265/RAF-265 & FLT3 & 1400 & Staurosporine & Erk7 & 35 \\
\hline CHIR-265/RAF-265 & FLT4 & 2000 & Staurosporine & FAK & 64 \\
\hline CHIR-265/RAF-265 & FMS & 250 & Staurosporine & FER & 24 \\
\hline CHIR-265/RAF-265 & FRK & 590 & Staurosporine & FES & 23 \\
\hline CHIR-265/RAF-265 & FYN & 2100 & Staurosporine & FGFR1 & 90 \\
\hline CHIR-265/RAF-265 & GCN2 & 55 & Staurosporine & FGFR2 & 100 \\
\hline CHIR-265/RAF-265 & $\mathrm{HCK}$ & 1200 & Staurosporine & FGFR3 & 88 \\
\hline CHIR-265/RAF-265 & HGK & 1300 & Staurosporine & FGFR4 & 250 \\
\hline CHIR-265/RAF-265 & $\mathrm{HH} 498$ & 5900 & Staurosporine & FGR & 17 \\
\hline CHIR-265/RAF-265 & KDR & 1300 & Staurosporine & FLT1 & 150 \\
\hline CHIR-265/RAF-265 & KHS1 & 830 & Staurosporine & FLT3 & 2.9 \\
\hline CHIR-265/RAF-265 & KIT & 200 & Staurosporine & FLT4 & 28 \\
\hline CHIR-265/RAF-265 & LCK & 640 & Staurosporine & FMS & 12 \\
\hline CHIR-265/RAF-265 & LOK & 60 & Staurosporine & FRK & 45 \\
\hline CHIR-265/RAF-265 & LYN & 370 & Staurosporine & Fused & 6700 \\
\hline CHIR-265/RAF-265 & NEK5 & 3200 & Staurosporine & FYN & 33 \\
\hline CHIR-265/RAF-265 & PDGFRa & 1100 & Staurosporine & GAK & 17 \\
\hline CHIR-265/RAF-265 & PDGFRb & 240 & Staurosporine & GCN2 & 16 \\
\hline CHIR-265/RAF-265 & PKR & 950 & Staurosporine & GSK3A & 140 \\
\hline CHIR-265/RAF-265 & RAF1 & 390 & Staurosporine & GSK3B & 69 \\
\hline CHIR-265/RAF-265 & RET & 150 & Staurosporine & $\mathrm{HCK}$ & 20 \\
\hline CHIR-265/RAF-265 & RIPK2 & 950 & Staurosporine & HGK & 24 \\
\hline CHIR-265/RAF-265 & SLK & 510 & Staurosporine & $\mathrm{HH} 498$ & 1000 \\
\hline
\end{tabular}




\begin{tabular}{|c|c|c|c|c|c|}
\hline CHIR-265/RAF-265 & SRC & 1100 & Staurosporine & HPK1 & 4.3 \\
\hline CHIR-265/RAF-265 & TIE1 & 150 & Staurosporine & IGF1R & 210 \\
\hline CHIR-265/RAF-265 & TIE2 & 1300 & Staurosporine & IKKe & 5.1 \\
\hline CHIR-265/RAF-265 & TNIK & 1500 & Staurosporine & INSR & 110 \\
\hline CHIR-265/RAF-265 & YES & 940 & Staurosporine & IRAK3 & 13 \\
\hline CHIR-265/RAF-265 & ZAK & 63 & Staurosporine & IRR & 81 \\
\hline $\mathrm{Cl}-1033$ & ABL1 & 1400 & Staurosporine & ITK & 19 \\
\hline $\mathrm{Cl}-1033$ & ABL2 & 870 & Staurosporine & JAK2 & 11 \\
\hline $\mathrm{Cl}-1033$ & ADCK3 & 1500 & Staurosporine & JAK3 & 10 \\
\hline $\mathrm{Cl}-1033$ & ADCK4 & 3400 & Staurosporine & JNK1 & 220 \\
\hline $\mathrm{Cl}-1033$ & ALK2 & 1700 & Staurosporine & JNK3 & 110 \\
\hline $\mathrm{Cl}-1033$ & AurB & 4200 & Staurosporine & KDR & 220 \\
\hline $\mathrm{Cl}-1033$ & AurC & 2800 & Staurosporine & KHS1 & 18 \\
\hline $\mathrm{Cl}-1033$ & $A X L$ & 5700 & Staurosporine & KHS2 & 8.2 \\
\hline $\mathrm{Cl}-1033$ & BIKE & 3800 & Staurosporine & KIT & 19 \\
\hline $\mathrm{Cl}-1033$ & BLK & 45 & Staurosporine & LATS1 & 42 \\
\hline $\mathrm{Cl}-1033$ & BMX & 2600 & Staurosporine & LATS2 & 17 \\
\hline $\mathrm{Cl}-1033$ & BRK & 3800 & Staurosporine & LCK & 30 \\
\hline $\mathrm{Cl}-1033$ & BTK & 1600 & Staurosporine & LIMK1 & 480 \\
\hline $\mathrm{Cl}-1033$ & CRIK & 1300 & Staurosporine & LIMK2 & 9200 \\
\hline $\mathrm{Cl}-1033$ & CSK & 6100 & Staurosporine & LKB1 & 61 \\
\hline $\mathrm{Cl}-1033$ & DDR1 & 400 & Staurosporine & LOK & 0.037 \\
\hline $\mathrm{Cl}-1033$ & EGFR & 0.19 & Staurosporine & LTK & 37 \\
\hline $\mathrm{Cl}-1033$ & EphA1 & 2500 & Staurosporine & LYN & 40 \\
\hline $\mathrm{Cl}-1033$ & EphA3 & 2100 & Staurosporine & MAP2K1 & 21 \\
\hline $\mathrm{Cl}-1033$ & EphA4 & 3600 & Staurosporine & MAP2K2 & 28 \\
\hline $\mathrm{Cl}-1033$ & EphA5 & 1200 & Staurosporine & MAP2K3 & 5 \\
\hline $\mathrm{Cl}-1033$ & EphA6 & 270 & Staurosporine & MAP2K4 & 70 \\
\hline $\mathrm{Cl}-1033$ & EphA8 & 2000 & Staurosporine & MAP2K6 & 3.4 \\
\hline $\mathrm{Cl}-1033$ & EphB2 & 2700 & Staurosporine & MAP3K4 & 1200 \\
\hline $\mathrm{Cl}-1033$ & EphB4 & 1800 & Staurosporine & MAP3K5 & 110 \\
\hline $\mathrm{Cl}-1033$ & ErbB2 & 87 & Staurosporine & MAPKAPK2 & 880 \\
\hline $\mathrm{Cl}-1033$ & ErbB4 & 29 & Staurosporine & MARK1 & 4 \\
\hline $\mathrm{Cl}-1033$ & ErK3 & 1700 & Staurosporine & MARK2 & 0.7 \\
\hline $\mathrm{Cl}-1033$ & FGR & 2800 & Staurosporine & MARK3 & 3 \\
\hline $\mathrm{Cl}-1033$ & FLT3 & 2700 & Staurosporine & MARK4 & 5.4 \\
\hline $\mathrm{Cl}-1033$ & FRK & 3400 & Staurosporine & MELK & 33 \\
\hline $\mathrm{Cl}-1033$ & Fused & 4500 & Staurosporine & MER & 6.4 \\
\hline $\mathrm{Cl}-1033$ & GAK & 100 & Staurosporine & MET & 190 \\
\hline $\mathrm{Cl}-1033$ & $\mathrm{HCK}$ & 4200 & Staurosporine & MLK1 & 10 \\
\hline $\mathrm{Cl}-1033$ & $\mathrm{HH} 498$ & 5600 & Staurosporine & MLK2 & 52 \\
\hline $\mathrm{Cl}-1033$ & IRAK3 & 3000 & Staurosporine & MLK3 & 20 \\
\hline $\mathrm{Cl}-1033$ & ITK & 5600 & Staurosporine & MNK1 & 170 \\
\hline $\mathrm{Cl}-1033$ & JAK3 & 630 & Staurosporine & MNK2 & 17 \\
\hline
\end{tabular}




\begin{tabular}{|c|c|c|c|c|c|}
\hline $\mathrm{Cl}-1033$ & JNK3 & 5500 & Staurosporine & MPSK1 & 270 \\
\hline $\mathrm{Cl}-1033$ & $\mathrm{KHS} 1$ & 2600 & Staurosporine & MRCKa & 57 \\
\hline $\mathrm{Cl}-1033$ & KIT & 7800 & Staurosporine & MRCKb & 42 \\
\hline $\mathrm{Cl}-1033$ & LCK & 320 & Staurosporine & MST1 & 0.19 \\
\hline $\mathrm{Cl}-1033$ & LOK & 1900 & Staurosporine & MST2 & 0.18 \\
\hline $\mathrm{Cl}-1033$ & LYN & 810 & Staurosporine & MST3 & 120 \\
\hline $\mathrm{Cl}-1033$ & MAP2K1 & 1800 & Staurosporine & MST4 & 140 \\
\hline $\mathrm{Cl}-1033$ & MAP2K2 & 1600 & Staurosporine & MUSK & 86 \\
\hline $\mathrm{Cl}-1033$ & MAP3K4 & 8400 & Staurosporine & MYO3A & 500 \\
\hline $\mathrm{Cl}-1033$ & MET & 5600 & Staurosporine & MYO3B & 300 \\
\hline $\mathrm{Cl}-1033$ & MNK1 & 260 & Staurosporine & NDR2 & 28 \\
\hline $\mathrm{Cl}-1033$ & MNK2 & 1800 & Staurosporine & NEK1 & 860 \\
\hline $\mathrm{Cl}-1033$ & PDGFRa & 5200 & Staurosporine & NEK2 & 750 \\
\hline $\mathrm{Cl}-1033$ & PDGFRb & 7500 & Staurosporine & NEK5 & 810 \\
\hline $\mathrm{Cl}-1033$ & QIK & 4900 & Staurosporine & NEK7 & 4500 \\
\hline $\mathrm{Cl}-1033$ & RET & 4200 & Staurosporine & NEK9 & 3100 \\
\hline $\mathrm{Cl}-1033$ & RIPK2 & 300 & Staurosporine & NLK & 220 \\
\hline $\mathrm{Cl}-1033$ & SLK & 1800 & Staurosporine & NuaK1 & 4.4 \\
\hline $\mathrm{Cl}-1033$ & SRC & 1100 & Staurosporine & p38g & 42 \\
\hline $\mathrm{Cl}-1033$ & SRM & 6700 & Staurosporine & PAK1 & 0.57 \\
\hline $\mathrm{Cl}-1033$ & TEC & 1500 & Staurosporine & PAK2 & 3.1 \\
\hline $\mathrm{Cl}-1033$ & TESK1 & 3200 & Staurosporine & PAK3 & 7.1 \\
\hline $\mathrm{Cl}-1033$ & TGFbR1 & 9600 & Staurosporine & PAK4 & 6.3 \\
\hline $\mathrm{Cl}-1033$ & TGFbR2 & 800 & Staurosporine & PAK5 & 1.2 \\
\hline $\mathrm{Cl}-1033$ & TIE1 & 2200 & Staurosporine & PAK6 & 0.57 \\
\hline $\mathrm{Cl}-1033$ & TXK & 700 & Staurosporine & PCTAIRE1 & 24 \\
\hline $\mathrm{Cl}-1033$ & TYRO3 & 1000 & Staurosporine & PCTAIRE2 & 96 \\
\hline $\mathrm{Cl}-1033$ & YES & 1600 & Staurosporine & PCTAIRE3 & 270 \\
\hline $\mathrm{Cl}-1033$ & ZAK & 1200 & Staurosporine & PDGFRa & 10 \\
\hline CР-690550 & CaMK1a & 5000 & Staurosporine & PDGFRb & 1.8 \\
\hline CP-690550 & DCAMKL3 & 4.5 & Staurosporine & PDK1 & 1.7 \\
\hline СР-690550 & JAK2 & 5 & Staurosporine & PFTAIRE1 & 160 \\
\hline CP-690550 & JAK3 & 2.2 & Staurosporine & PHKg1 & 0.65 \\
\hline CP-690550 & LCK & 1800 & Staurosporine & PHKg2 & 0.14 \\
\hline CP-690550 & MST2 & 4300 & Staurosporine & PIM1 & 3.2 \\
\hline CP-690550 & PKN1 & 200 & Staurosporine & PIM2 & 1.9 \\
\hline CP-690550 & SNRK & 420 & Staurosporine & PIM3 & 0.51 \\
\hline CP-690550 & TNK1 & 640 & Staurosporine & PIP5K1A & 43 \\
\hline CР-690550 & TYK2 & 620 & Staurosporine & PIP5K2B & 77 \\
\hline CP-724714 & CDK11 & 1100 & Staurosporine & PKACa & 19 \\
\hline CP-724714 & CDK8 & 2300 & Staurosporine & PKACb & 29 \\
\hline CP-724714 & EGFR & 42 & Staurosporine & PKCd & 1.5 \\
\hline CP-724714 & ErbB2 & 43 & Staurosporine & $\mathrm{PKCe}$ & 0.25 \\
\hline CP-724714 & ErbB4 & 260 & Staurosporine & PKCh & 4.8 \\
\hline
\end{tabular}




\begin{tabular}{|c|c|c|c|c|c|}
\hline CP-724714 & MNK2 & 840 & Staurosporine & $\mathrm{PKCt}$ & 8.5 \\
\hline Dasatinib & ABL1 & 0.53 & Staurosporine & PKD1 & 27 \\
\hline Dasatinib & ABL2 & 0.17 & Staurosporine & PKD2 & 72 \\
\hline Dasatinib & ACK & 5.6 & Staurosporine & PKD3 & 18 \\
\hline Dasatinib & ACTR2 & 210 & Staurosporine & PKG1 & 32 \\
\hline Dasatinib & ACTR2B & 570 & Staurosporine & PKG2 & 16 \\
\hline Dasatinib & ADCK3 & 190 & Staurosporine & PKN1 & 1.3 \\
\hline Dasatinib & ALK1 & 460 & Staurosporine & PKN2 & 2.5 \\
\hline Dasatinib & ALK2 & 620 & Staurosporine & PKR & 360 \\
\hline Dasatinib & ALK4 & 330 & Staurosporine & PLK1 & 190 \\
\hline Dasatinib & BLK & 0.21 & Staurosporine & PLK3 & 910 \\
\hline Dasatinib & BMX & 1.4 & Staurosporine & PLK4 & 0.77 \\
\hline Dasatinib & BRAF & 500 & Staurosporine & PRKX & 13 \\
\hline Dasatinib & BRK & 7.8 & Staurosporine & PYK2 & 3.5 \\
\hline Dasatinib & BTK & 1.4 & Staurosporine & QIK & 1.1 \\
\hline Dasatinib & CK1e & 1500 & Staurosporine & RET & 15 \\
\hline Dasatinib & CSK & 1 & Staurosporine & RIOK1 & 130 \\
\hline Dasatinib & DDR1 & 0.69 & Staurosporine & RIOK3 & 76 \\
\hline Dasatinib & DDR2 & 3.2 & Staurosporine & RIPK2 & 5200 \\
\hline Dasatinib & DMPK1 & 1300 & Staurosporine & ROS & 30 \\
\hline Dasatinib & DMPK2 & 1200 & Staurosporine & SgK085 & 470 \\
\hline Dasatinib & EGFR & 120 & Staurosporine & SgK288 & 270 \\
\hline Dasatinib & EphA1 & 4.1 & Staurosporine & SIK & 2.2 \\
\hline Dasatinib & EphA2 & 0.85 & Staurosporine & skMLCK & 61 \\
\hline Dasatinib & EphA3 & 0.093 & Staurosporine & SLK & 0.024 \\
\hline Dasatinib & EphA4 & 1.2 & Staurosporine & smMLCK & 15 \\
\hline Dasatinib & EphA5 & 0.24 & Staurosporine & SNRK & 0.086 \\
\hline Dasatinib & EphA8 & 0.24 & Staurosporine & SRC & 86 \\
\hline Dasatinib & EphB1 & 0.45 & Staurosporine & SRM & 740 \\
\hline Dasatinib & EphB2 & 0.39 & Staurosporine & SRPK1 & 5.5 \\
\hline Dasatinib & EphB3 & 6.9 & Staurosporine & SRPK2 & 67 \\
\hline Dasatinib & EphB4 & 0.34 & Staurosporine & STK33 & 1.8 \\
\hline Dasatinib & ErbB2 & 1400 & Staurosporine & SYK & 14 \\
\hline Dasatinib & ErbB4 & 55 & Staurosporine & TEC & 1300 \\
\hline Dasatinib & FGFR1 & 3700 & Staurosporine & TESK1 & 320 \\
\hline Dasatinib & FGFR2 & 1400 & Staurosporine & TGFbR1 & 2000 \\
\hline Dasatinib & FGR & 0.5 & Staurosporine & TIE1 & 65 \\
\hline Dasatinib & FLT1 & 5000 & Staurosporine & TIE2 & 140 \\
\hline Dasatinib & FMS & 0.58 & Staurosporine & TLK1 & 27 \\
\hline Dasatinib & FRK & 0.31 & Staurosporine & TLK2 & 16 \\
\hline Dasatinib & Fused & 210 & Staurosporine & TNIK & 4.7 \\
\hline Dasatinib & FYN & 0.79 & Staurosporine & TNK1 & 2.5 \\
\hline Dasatinib & GAK & 2.6 & Staurosporine & TRKA & 4 \\
\hline Dasatinib & GCN2 & 1600 & Staurosporine & TRKB & 3.8 \\
\hline
\end{tabular}




\begin{tabular}{|c|c|c|c|c|c|}
\hline Dasatinib & HCK & 0.35 & Staurosporine & TRKC & 17 \\
\hline Dasatinib & HGK & 3100 & Staurosporine & TSSK1 & 24 \\
\hline Dasatinib & $\mathrm{HH} 498$ & 11 & Staurosporine & TTK & 61 \\
\hline Dasatinib & HPK1 & 980 & Staurosporine & TXK & 230 \\
\hline Dasatinib & JAK2 & 1000 & Staurosporine & TYK2 & 56 \\
\hline Dasatinib & KHS1 & 45 & Staurosporine & TYRO3 & 280 \\
\hline Dasatinib & KHS2 & 640 & Staurosporine & Wee1 & 1600 \\
\hline Dasatinib & KIT & 0.62 & Staurosporine & YANK2 & 98 \\
\hline Dasatinib & LCK & 0.2 & Staurosporine & YANK3 & 310 \\
\hline Dasatinib & LIMK1 & 570 & Staurosporine & YES & 52 \\
\hline Dasatinib & LIMK2 & 86 & Staurosporine & YSK1 & 110 \\
\hline Dasatinib & LOK & 1200 & Staurosporine & ZAP70 & 44 \\
\hline Dasatinib & LYN & 0.57 & SU-14813 & AAK1 & 66 \\
\hline Dasatinib & MAP2K1 & 1000 & SU-14813 & ABL1 & 1500 \\
\hline Dasatinib & MAP2K2 & 1400 & SU-14813 & ACK & 4900 \\
\hline Dasatinib & MAP3K4 & 310 & SU-14813 & ALK & 490 \\
\hline Dasatinib & MRCKa & 2000 & SU-14813 & AMPKa1 & 100 \\
\hline Dasatinib & MRCKb & 2100 & SU-14813 & AMPKa2 & 290 \\
\hline Dasatinib & MST4 & 1900 & SU-14813 & AurB & 340 \\
\hline Dasatinib & MYT1 & 130 & SU-14813 & AurC & 810 \\
\hline Dasatinib & NLK & 260 & SU-14813 & $A X L$ & 84 \\
\hline Dasatinib & p38a & 27 & SU-14813 & BIKE & 27 \\
\hline Dasatinib & p38b & 410 & SU-14813 & BLK & 750 \\
\hline Dasatinib & PDGFRa & 0.47 & SU-14813 & BMPR2 & 200 \\
\hline Dasatinib & PDGFRb & 0.63 & SU-14813 & BRSK1 & 5000 \\
\hline Dasatinib & QIK & 6.4 & SU-14813 & BRSK2 & 4100 \\
\hline Dasatinib & RAF1 & 570 & SU-14813 & CaMK1a & 3300 \\
\hline Dasatinib & RET & 730 & SU-14813 & CaMK1d & 970 \\
\hline Dasatinib & RIPK2 & 31 & SU-14813 & CaMK1g & 940 \\
\hline Dasatinib & SIK & 3.9 & SU-14813 & CaMK2a & 350 \\
\hline Dasatinib & SLK & 720 & SU-14813 & CaMK2b & 2300 \\
\hline Dasatinib & SRC & 0.21 & SU-14813 & CaMK2d & 760 \\
\hline Dasatinib & SRM & 13 & SU-14813 & CaMK2g & 1100 \\
\hline Dasatinib & SYK & 2900 & SU-14813 & CaMK4 & 2700 \\
\hline Dasatinib & TEC & 13 & SU-14813 & CaMKK1 & 850 \\
\hline Dasatinib & TESK1 & 33 & SU-14813 & CaMKK2 & 2500 \\
\hline Dasatinib & TGFbR1 & 230 & SU-14813 & CaMLCK & 42 \\
\hline Dasatinib & TGFbR2 & 2900 & SU-14813 & CDK7 & 930 \\
\hline Dasatinib & TNIK & 2000 & SU-14813 & $\mathrm{CHK} 1$ & 1200 \\
\hline Dasatinib & TXK & 2.1 & SU-14813 & CK1a & 1500 \\
\hline Dasatinib & Wee1 & 7000 & SU-14813 & CK1d & 260 \\
\hline Dasatinib & YES & 0.3 & SU-14813 & CK1e & 340 \\
\hline Dasatinib & ZAK & 45 & SU-14813 & CK1g1 & 1000 \\
\hline EKB-569 & AAK1 & 3500 & SU-14813 & CK1g2 & 790 \\
\hline
\end{tabular}




\begin{tabular}{|c|c|c|c|c|c|}
\hline EKB-569 & ABL1 & 560 & SU-14813 & CK1g3 & 570 \\
\hline EKB-569 & ABL2 & 370 & SU-14813 & CK2a1 & 97 \\
\hline EKB-569 & ACK & 5200 & SU-14813 & CK2a2 & 140 \\
\hline EKB-569 & ADCK4 & 6100 & SU-14813 & CLK1 & 360 \\
\hline EKB-569 & ALK & 7100 & SU-14813 & CLK2 & 150 \\
\hline EKB-569 & AXL & 920 & SU-14813 & CLK4 & 250 \\
\hline EKB-569 & BIKE & 2200 & SU-14813 & CRIK & 94 \\
\hline EKB-569 & BLK & 78 & SU-14813 & DAPK1 & 270 \\
\hline EKB-569 & BMX & 7400 & SU-14813 & DAPK2 & 400 \\
\hline EKB-569 & BTK & 4900 & SU-14813 & DAPK3 & 170 \\
\hline EKB-569 & CaMK1d & 5900 & SU-14813 & DCAMKL1 & 550 \\
\hline EKB-569 & CaMKK1 & 5100 & SU-14813 & DCAMKL3 & 240 \\
\hline EKB-569 & CaMKK2 & 2500 & SU-14813 & DLK & 560 \\
\hline EKB-569 & $\mathrm{CHK} 1$ & 1900 & SU-14813 & DRAK1 & 24 \\
\hline EKB-569 & CK1a & 1000 & SU-14813 & DRAK2 & 320 \\
\hline EKB-569 & CK1d & 1100 & SU-14813 & DYRK1B & 2600 \\
\hline EKB-569 & CK1e & 100 & SU-14813 & EphA6 & 2000 \\
\hline EKB-569 & CLK1 & 3300 & SU-14813 & FAK & 1200 \\
\hline EKB-569 & CLK2 & 1800 & SU-14813 & FER & 34 \\
\hline EKB-569 & CLK3 & 5600 & SU-14813 & FGFR1 & 1900 \\
\hline EKB-569 & CLK4 & 3900 & SU-14813 & FGFR2 & 790 \\
\hline EKB-569 & CRIK & 310 & SU-14813 & FGFR3 & 560 \\
\hline EKB-569 & CSK & 3400 & SU-14813 & FGR & 390 \\
\hline EKB-569 & DCAMKL3 & 4900 & SU-14813 & FLT1 & 4.7 \\
\hline EKB-569 & DLK & 2600 & SU-14813 & FLT3 & 0.54 \\
\hline EKB-569 & DMPK1 & 59 & SU-14813 & FLT4 & 50 \\
\hline EKB-569 & DRAK1 & 200 & SU-14813 & FMS & 3.6 \\
\hline EKB-569 & DRAK2 & 4200 & SU-14813 & FYN & 2600 \\
\hline EKB-569 & DYRK1B & 4000 & SU-14813 & GAK & 44 \\
\hline EKB-569 & EGFR & 0.44 & SU-14813 & GCN2 & 470 \\
\hline EKB-569 & EphA3 & 4000 & SU-14813 & $\mathrm{HCK}$ & 2200 \\
\hline EKB-569 & EphA8 & 3300 & SU-14813 & HGK & 260 \\
\hline EKB-569 & EphB4 & 2800 & SU-14813 & HPK1 & 15 \\
\hline EKB-569 & ErbB2 & 500 & SU-14813 & IKKe & 480 \\
\hline EKB-569 & ErbB4 & 21 & SU-14813 & INSR & 1200 \\
\hline EKB-569 & FER & 8800 & SU-14813 & IRAK3 & 1900 \\
\hline EKB-569 & FES & 5700 & SU-14813 & IRR & 2000 \\
\hline EKB-569 & FGR & 950 & SU-14813 & ITK & 190 \\
\hline EKB-569 & FMS & 4300 & SU-14813 & JAK2 & 1600 \\
\hline EKB-569 & FRK & 680 & SU-14813 & JAK3 & 580 \\
\hline EKB-569 & Fused & 4000 & SU-14813 & KDR & 2.3 \\
\hline EKB-569 & FYN & 2400 & SU-14813 & KHS1 & 300 \\
\hline EKB-569 & GAK & 6.4 & SU-14813 & KHS2 & 210 \\
\hline EKB-569 & GCN2 & 290 & SU-14813 & KIT & 0.68 \\
\hline
\end{tabular}




\begin{tabular}{|c|c|c|c|c|c|}
\hline EKB-569 & $\mathrm{HCK}$ & 2300 & SU-14813 & LATS1 & 850 \\
\hline EKB-569 & HGK & 330 & SU-14813 & LATS2 & 430 \\
\hline EKB-569 & $\mathrm{HH} 498$ & 7200 & SU-14813 & LCK & 2300 \\
\hline EKB-569 & HPK1 & 270 & SU-14813 & LKB1 & 360 \\
\hline EKB-569 & ITK & 2500 & SU-14813 & LOK & 230 \\
\hline EKB-569 & JAK3 & 25 & SU-14813 & LTK & 4200 \\
\hline EKB-569 & KHS1 & 10 & SU-14813 & LYN & 810 \\
\hline EKB-569 & KHS2 & 170 & SU-14813 & MAP2K1 & 77 \\
\hline EKB-569 & LCK & 99 & SU-14813 & MAP2K2 & 120 \\
\hline EKB-569 & LOK & 330 & SU-14813 & MAP2K4 & 1400 \\
\hline EKB-569 & LYN & 720 & SU-14813 & MAP2K6 & 5500 \\
\hline EKB-569 & MAP2K1 & 360 & SU-14813 & MARK1 & 1500 \\
\hline EKB-569 & MAP2K2 & 810 & SU-14813 & MARK2 & 440 \\
\hline EKB-569 & MAP3K4 & 280 & SU-14813 & MARK3 & 1100 \\
\hline EKB-569 & MARK1 & 4000 & SU-14813 & MARK4 & 2000 \\
\hline EKB-569 & MER & 1300 & SU-14813 & MELK & 340 \\
\hline EKB-569 & MET & 6200 & SU-14813 & MER & 66 \\
\hline EKB-569 & MST1 & 3600 & SU-14813 & MLK1 & 2600 \\
\hline EKB-569 & MST2 & 2400 & SU-14813 & MLK3 & 1700 \\
\hline EKB-569 & MST3 & 2100 & SU-14813 & MNK2 & 5500 \\
\hline EKB-569 & MYT1 & 2900 & SU-14813 & MPSK1 & 730 \\
\hline EKB-569 & NEK1 & 3900 & SU-14813 & MST1 & 130 \\
\hline EKB-569 & NEK2 & 680 & SU-14813 & MST2 & 65 \\
\hline EKB-569 & PAK1 & 3400 & SU-14813 & MST3 & 100 \\
\hline EKB-569 & PHKg1 & 3500 & SU-14813 & MST4 & 330 \\
\hline EKB-569 & PIP5K2B & 2600 & SU-14813 & MUSK & 100 \\
\hline EKB-569 & PKCd & 4600 & SU-14813 & NDR2 & 2000 \\
\hline EKB-569 & PKR & 1900 & SU-14813 & NEK2 & 1100 \\
\hline EKB-569 & QIK & 4500 & SU-14813 & NEK7 & 3200 \\
\hline EKB-569 & skMLCK & 3900 & SU-14813 & NuaK1 & 460 \\
\hline EKB-569 & SLK & 360 & SU-14813 & PAK3 & 190 \\
\hline EKB-569 & SNRK & 2700 & SU-14813 & PCTAIRE1 & 940 \\
\hline EKB-569 & $\mathrm{SRC}$ & 280 & SU-14813 & PDGFRa & 1.1 \\
\hline EKB-569 & STK33 & 4500 & SU-14813 & PDGFRb & 0.29 \\
\hline EKB-569 & SYK & 7200 & SU-14813 & PDK1 & 2600 \\
\hline EKB-569 & TIE1 & 1000 & SU-14813 & PHKg1 & 83 \\
\hline EKB-569 & TNIK & 170 & SU-14813 & PHKg2 & 100 \\
\hline EKB-569 & TXK & 5500 & SU-14813 & PIM3 & 2400 \\
\hline EKB-569 & TYRO3 & 3700 & SU-14813 & PIP5K1A & 130 \\
\hline EKB-569 & Wee1 & 770 & SU-14813 & PIP5K2B & 260 \\
\hline EKB-569 & YANK2 & 6000 & SU-14813 & PKCt & 7200 \\
\hline EKB-569 & YES & 1100 & SU-14813 & PKD1 & 2400 \\
\hline EKB-569 & YSK1 & 3400 & SU-14813 & PKD2 & 3700 \\
\hline EKB-569 & ZAK & 1200 & SU-14813 & PKD3 & 2700 \\
\hline
\end{tabular}




\begin{tabular}{|c|c|c|c|c|c|}
\hline Erlotinib & AAK1 & 1200 & SU-14813 & PKN1 & 1900 \\
\hline Erlotinib & ABL1 & 310 & SU-14813 & PKN2 & 3800 \\
\hline Erlotinib & ABL2 & 200 & SU-14813 & PKR & 1300 \\
\hline Erlotinib & ADCK3 & 1900 & SU-14813 & PLK4 & 180 \\
\hline Erlotinib & ADCK4 & 2500 & SU-14813 & PYK2 & 600 \\
\hline Erlotinib & ALK & 1200 & SU-14813 & QIK & 750 \\
\hline Erlotinib & AurA & 2200 & SU-14813 & RET & 37 \\
\hline Erlotinib & AurB & 1400 & SU-14813 & RIOK1 & 590 \\
\hline Erlotinib & AurC & 600 & SU-14813 & RIOK3 & 9700 \\
\hline Erlotinib & BIKE & 1200 & SU-14813 & RIPK1 & 1200 \\
\hline Erlotinib & BLK & 190 & SU-14813 & SgK085 & 32 \\
\hline Erlotinib & CK1d & 3500 & SU-14813 & SgK288 & 160 \\
\hline Erlotinib & CRIK & 680 & SU-14813 & SIK & 2700 \\
\hline Erlotinib & DDR1 & 790 & SU-14813 & skMLCK & 190 \\
\hline Erlotinib & DMPK1 & 2900 & SU-14813 & SLK & 460 \\
\hline Erlotinib & DMPK2 & 3400 & SU-14813 & smMLCK & 360 \\
\hline Erlotinib & EGFR & 0.67 & SU-14813 & SNRK & 400 \\
\hline Erlotinib & EphA3 & 2400 & SU-14813 & SRC & 4900 \\
\hline Erlotinib & EphA6 & 440 & SU-14813 & SRM & 62 \\
\hline Erlotinib & EphA7 & 1400 & SU-14813 & SRPK1 & 4000 \\
\hline Erlotinib & EphA8 & 940 & SU-14813 & SRPK2 & 200 \\
\hline Erlotinib & ErbB4 & 230 & SU-14813 & STK33 & 130 \\
\hline Erlotinib & ErK4 & 2500 & SU-14813 & TLK1 & 630 \\
\hline Erlotinib & FGR & 1100 & SU-14813 & TLK2 & 270 \\
\hline Erlotinib & FLT1 & 4400 & SU-14813 & TNIK & 90 \\
\hline Erlotinib & FLT4 & 2100 & SU-14813 & TNK1 & 1500 \\
\hline Erlotinib & FRK & 2000 & SU-14813 & TRKA & 480 \\
\hline Erlotinib & GAK & 3.1 & SU-14813 & TRKB & 990 \\
\hline Erlotinib & GCN2 & 4400 & SU-14813 & TRKC & 220 \\
\hline Erlotinib & HCK & 1800 & SU-14813 & TTK & 180 \\
\hline Erlotinib & $\mathrm{HH} 498$ & 570 & SU-14813 & TYK2 & 5100 \\
\hline Erlotinib & JAK3 & 930 & SU-14813 & TYRO3 & 2400 \\
\hline Erlotinib & LCK & 250 & SU-14813 & Wee1 & 3000 \\
\hline Erlotinib & LOK & 19 & SU-14813 & YES & 260 \\
\hline Erlotinib & LTK & 890 & SU-14813 & YSK1 & 360 \\
\hline Erlotinib & LYN & 530 & Sunitinib & AAK1 & 11 \\
\hline Erlotinib & MET & 3800 & Sunitinib & ABL1 & 830 \\
\hline Erlotinib & MNK1 & 690 & Sunitinib & ABL2 & 1000 \\
\hline Erlotinib & PDGFRa & 1800 & Sunitinib & ACK & 8900 \\
\hline Erlotinib & PDGFRb & 1400 & Sunitinib & AKT2 & 2700 \\
\hline Erlotinib & PKR & 1300 & Sunitinib & ALK & 170 \\
\hline Erlotinib & RET & 1300 & Sunitinib & AMPKa1 & 19 \\
\hline Erlotinib & RIPK2 & 680 & Sunitinib & AMPKa2 & 89 \\
\hline Erlotinib & skMLCK & 970 & Sunitinib & AurA & 1700 \\
\hline
\end{tabular}




\begin{tabular}{|c|c|c|c|c|c|}
\hline Erlotinib & SLK & 26 & Sunitinib & AurB & 380 \\
\hline Erlotinib & SRC & 700 & Sunitinib & AurC & 220 \\
\hline Erlotinib & TIE1 & 850 & Sunitinib & $A X L$ & 9 \\
\hline Erlotinib & TNK1 & 630 & Sunitinib & BIKE & 5.5 \\
\hline Erlotinib & TYRO3 & 3900 & Sunitinib & BLK & 65 \\
\hline Erlotinib & YES & 2200 & Sunitinib & BMPR2 & 570 \\
\hline Flavopiridol & AAK1 & 5300 & Sunitinib & BRSK1 & 3500 \\
\hline Flavopiridol & ACTR2B & 2400 & Sunitinib & BRSK2 & 1100 \\
\hline Flavopiridol & ALK & 670 & Sunitinib & CaMK1a & 970 \\
\hline Flavopiridol & ALK2 & 1900 & Sunitinib & CaMK1d & 510 \\
\hline Flavopiridol & $A X L$ & 3500 & Sunitinib & CaMK1g & 440 \\
\hline Flavopiridol & CaMK2a & 1700 & Sunitinib & CaMK2a & 80 \\
\hline Flavopiridol & CaMK4 & 3200 & Sunitinib & CaMK2b & 1400 \\
\hline Flavopiridol & CaMKK1 & 79 & Sunitinib & CaMK2d & 420 \\
\hline Flavopiridol & CaMKK2 & 430 & Sunitinib & CaMK2g & 690 \\
\hline Flavopiridol & CDK11 & 57 & Sunitinib & CaMK4 & 890 \\
\hline Flavopiridol & CDK2 & 550 & Sunitinib & CaMKK1 & 420 \\
\hline Flavopiridol & CDK3 & 410 & Sunitinib & CaMKK2 & 1500 \\
\hline Flavopiridol & CDK5 & 110 & Sunitinib & CaMLCK & 23 \\
\hline Flavopiridol & CDK7 & 23 & Sunitinib & CDK7 & 330 \\
\hline Flavopiridol & CDK8 & 120 & Sunitinib & $\mathrm{CHK} 1$ & 300 \\
\hline Flavopiridol & CDK9 & 6.4 & Sunitinib & CK1a & 550 \\
\hline Flavopiridol & CLK1 & 1700 & Sunitinib & CK1d & 15 \\
\hline Flavopiridol & CLK2 & 2200 & Sunitinib & CK1e & 13 \\
\hline Flavopiridol & CLK3 & 1600 & Sunitinib & CK1g1 & 930 \\
\hline Flavopiridol & CRIK & 110 & Sunitinib & CK1g2 & 110 \\
\hline Flavopiridol & DCAMKL1 & 2100 & Sunitinib & CK1g3 & 240 \\
\hline Flavopiridol & DCAMKL3 & 1100 & Sunitinib & CK2a1 & 81 \\
\hline Flavopiridol & DMPK1 & 650 & Sunitinib & CK2a2 & 370 \\
\hline Flavopiridol & DRAK2 & 1900 & Sunitinib & CLK1 & 22 \\
\hline Flavopiridol & DYRK1B & 84 & Sunitinib & CLK2 & 20 \\
\hline Flavopiridol & EphA2 & 2500 & Sunitinib & CLK4 & 29 \\
\hline Flavopiridol & EphA3 & 3300 & Sunitinib & CRIK & 3900 \\
\hline Flavopiridol & ErK5 & 620 & Sunitinib & DAPK1 & 120 \\
\hline Flavopiridol & Erk7 & 330 & Sunitinib & DAPK2 & 150 \\
\hline Flavopiridol & FMS & 2800 & Sunitinib & DAPK3 & 22 \\
\hline Flavopiridol & GAK & 370 & Sunitinib & DCAMKL1 & 370 \\
\hline Flavopiridol & GSK3A & 1300 & Sunitinib & DCAMKL2 & 2700 \\
\hline Flavopiridol & GSK3B & 730 & Sunitinib & DCAMKL3 & 110 \\
\hline Flavopiridol & $\mathrm{HH} 498$ & 55 & Sunitinib & DDR1 & 2000 \\
\hline Flavopiridol & HPK1 & 3300 & Sunitinib & DLK & 100 \\
\hline Flavopiridol & IKKe & 3100 & Sunitinib & DRAK1 & 1 \\
\hline Flavopiridol & JNK3 & 5500 & Sunitinib & DRAK2 & 110 \\
\hline Flavopiridol & KHS2 & 4500 & Sunitinib & DYRK1B & 2300 \\
\hline
\end{tabular}




\begin{tabular}{|c|c|c|c|c|c|}
\hline Flavopiridol & LTK & 2200 & Sunitinib & EphA3 & 2100 \\
\hline Flavopiridol & MER & 6800 & Sunitinib & EphA6 & 960 \\
\hline Flavopiridol & MLK1 & 5700 & Sunitinib & EphA7 & 2400 \\
\hline Flavopiridol & MLK2 & 5200 & Sunitinib & EphB1 & 480 \\
\hline Flavopiridol & MNK2 & 3200 & Sunitinib & FAK & 440 \\
\hline Flavopiridol & MRCKa & 9500 & Sunitinib & FER & 1100 \\
\hline Flavopiridol & MRCKb & 3300 & Sunitinib & FES & 960 \\
\hline Flavopiridol & MST2 & 9400 & Sunitinib & FGFR1 & 520 \\
\hline Flavopiridol & PCTAIRE1 & 440 & Sunitinib & FGFR2 & 570 \\
\hline Flavopiridol & PCTAIRE2 & 480 & Sunitinib & FGFR3 & 290 \\
\hline Flavopiridol & PCTAIRE3 & 1100 & Sunitinib & FGR & 270 \\
\hline Flavopiridol & PFTAIRE1 & 110 & Sunitinib & FLT1 & 1.8 \\
\hline Flavopiridol & PHKg1 & 2200 & Sunitinib & FLT3 & 0.47 \\
\hline Flavopiridol & PHKg2 & 2900 & Sunitinib & FLT4 & 50 \\
\hline Flavopiridol & PIM1 & 560 & Sunitinib & FMS & 2 \\
\hline Flavopiridol & PIM2 & 770 & Sunitinib & FRK & 530 \\
\hline Flavopiridol & PIM3 & 600 & Sunitinib & FYN & 520 \\
\hline Flavopiridol & PKCd & 590 & Sunitinib & GAK & 20 \\
\hline Flavopiridol & PKCe & 380 & Sunitinib & GCN2 & 180 \\
\hline Flavopiridol & PKCh & 350 & Sunitinib & $\mathrm{HCK}$ & 880 \\
\hline Flavopiridol & PKCt & 350 & Sunitinib & HGK & 140 \\
\hline Flavopiridol & PKD1 & 520 & Sunitinib & HPK1 & 16 \\
\hline Flavopiridol & PKD2 & 1100 & Sunitinib & IGF1R & 2600 \\
\hline Flavopiridol & PKD3 & 170 & Sunitinib & IKKe & 620 \\
\hline Flavopiridol & PKN1 & 3500 & Sunitinib & INSR & 500 \\
\hline Flavopiridol & PKR & 5200 & Sunitinib & IRAK3 & 940 \\
\hline Flavopiridol & SNRK & 1400 & Sunitinib & IRR & 430 \\
\hline Flavopiridol & SRM & 1400 & Sunitinib & ITK & 13 \\
\hline Flavopiridol & STK33 & 1700 & Sunitinib & JAK2 & 410 \\
\hline Flavopiridol & TGFbR2 & 5600 & Sunitinib & JAK3 & 1200 \\
\hline Gefitinib & BLK & 1200 & Sunitinib & KDR & 1.5 \\
\hline Gefitinib & CK1e & 430 & Sunitinib & KHS1 & 41 \\
\hline Gefitinib & CRIK & 1300 & Sunitinib & KHS2 & 180 \\
\hline Gefitinib & DAPK3 & 5700 & Sunitinib & KIT & 0.37 \\
\hline Gefitinib & DMPK1 & 6900 & Sunitinib & LATS1 & 630 \\
\hline Gefitinib & DRAK1 & 2000 & Sunitinib & LATS2 & 460 \\
\hline Gefitinib & DRAK2 & 3800 & Sunitinib & LCK & 230 \\
\hline Gefitinib & EGFR & 1 & Sunitinib & LKB1 & 38 \\
\hline Gefitinib & EphA1 & 4000 & Sunitinib & LOK & 19 \\
\hline Gefitinib & EphA6 & 590 & Sunitinib & LTK & 1800 \\
\hline Gefitinib & EphA8 & 1800 & Sunitinib & LYN & 270 \\
\hline Gefitinib & ErbB2 & 3500 & Sunitinib & MAP2K1 & 130 \\
\hline Gefitinib & ErbB4 & 410 & Sunitinib & MAP2K2 & 110 \\
\hline Gefitinib & ErK3 & 1600 & Sunitinib & MARK1 & 1200 \\
\hline
\end{tabular}




\begin{tabular}{|c|c|c|c|c|c|}
\hline Gefitinib & ErK4 & 3100 & Sunitinib & MARK2 & 310 \\
\hline Gefitinib & FRK & 2000 & Sunitinib & MARK3 & 410 \\
\hline Gefitinib & GAK & 13 & Sunitinib & MARK4 & 3600 \\
\hline Gefitinib & HCK & 4400 & Sunitinib & MELK & 350 \\
\hline Gefitinib & IRAK3 & 1500 & Sunitinib & MER & 26 \\
\hline Gefitinib & JNK3 & 3200 & Sunitinib & MLK1 & 3400 \\
\hline Gefitinib & LCK & 630 & Sunitinib & MLK3 & 1300 \\
\hline Gefitinib & LOK & 470 & Sunitinib & MPSK1 & 250 \\
\hline Gefitinib & LYN & 990 & Sunitinib & MST1 & 19 \\
\hline Gefitinib & MNK1 & 290 & Sunitinib & MST2 & 56 \\
\hline Gefitinib & MNK2 & 1200 & Sunitinib & MST3 & 63 \\
\hline Gefitinib & PHKg1 & 3700 & Sunitinib & MST4 & 340 \\
\hline Gefitinib & PIM3 & 5800 & Sunitinib & MUSK & 490 \\
\hline Gefitinib & QIK & 2100 & Sunitinib & МYO3B & 4500 \\
\hline Gefitinib & RIPK2 & 530 & Sunitinib & NDR2 & 970 \\
\hline Gefitinib & skMLCK & 1900 & Sunitinib & NEK2 & 1400 \\
\hline Gefitinib & SLK & 920 & Sunitinib & NEK7 & 4100 \\
\hline Gefitinib & SRC & 3800 & Sunitinib & NuaK1 & 48 \\
\hline Gefitinib & TXK & 6000 & Sunitinib & PAK3 & 16 \\
\hline GW-2580 & FMS & 1.6 & Sunitinib & PAK4 & 2300 \\
\hline GW-2580 & TRKA & 630 & Sunitinib & PAK5 & 640 \\
\hline GW-2580 & TRKB & 36 & Sunitinib & PAK6 & 2400 \\
\hline GW-2580 & TRKC & 120 & Sunitinib & PCTAIRE1 & 150 \\
\hline GW-786034 & AAK1 & 2900 & Sunitinib & PCTAIRE2 & 1200 \\
\hline GW-786034 & ABL1 & 1700 & Sunitinib & PCTAIRE3 & 1700 \\
\hline GW-786034 & ABL2 & 3000 & Sunitinib & PDGFRa & 0.79 \\
\hline GW-786034 & ACTR2B & 2400 & Sunitinib & PDGFRb & 0.075 \\
\hline GW-786034 & ALK & 2200 & Sunitinib & PDK1 & 3500 \\
\hline GW-786034 & AurA & 7100 & Sunitinib & PFTAIRE1 & 270 \\
\hline GW-786034 & AurC & 750 & Sunitinib & PHKg1 & 5.5 \\
\hline GW-786034 & BIKE & 8800 & Sunitinib & PHKg2 & 5.9 \\
\hline GW-786034 & BLK & 2600 & Sunitinib & PIM3 & 2400 \\
\hline GW-786034 & BRAF & 730 & Sunitinib & PIP5K1A & 5400 \\
\hline GW-786034 & BRK & 2300 & Sunitinib & PIP5K2B & 39 \\
\hline GW-786034 & CaMK1a & 2100 & Sunitinib & $\mathrm{PKCt}$ & 4300 \\
\hline GW-786034 & CaMK1g & 3700 & Sunitinib & PKD1 & 310 \\
\hline GW-786034 & DDR1 & 57 & Sunitinib & PKD2 & 380 \\
\hline GW-786034 & DDR2 & 98 & Sunitinib & PKD3 & 280 \\
\hline GW-786034 & FER & 2700 & Sunitinib & PKN1 & 710 \\
\hline GW-786034 & FGFR1 & 990 & Sunitinib & PKN2 & 1300 \\
\hline GW-786034 & FGFR2 & 210 & Sunitinib & PKR & 670 \\
\hline GW-786034 & FGFR3 & 740 & Sunitinib & PLK4 & 190 \\
\hline GW-786034 & FGFR4 & 2800 & Sunitinib & PYK2 & 82 \\
\hline GW-786034 & FGR & 1600 & Sunitinib & QIK & 580 \\
\hline
\end{tabular}




\begin{tabular}{|c|c|c|c|c|c|}
\hline GW-786034 & FLT1 & 14 & Sunitinib & RET & 12 \\
\hline GW-786034 & FLT3 & 1100 & Sunitinib & RIOK1 & 35 \\
\hline GW-786034 & FLT4 & 27 & Sunitinib & RIOK3 & 3800 \\
\hline GW-786034 & FMS & 7.9 & Sunitinib & RIPK1 & 370 \\
\hline GW-786034 & FRK & 750 & Sunitinib & SgK085 & 15 \\
\hline GW-786034 & Fused & 470 & Sunitinib & SgK288 & 310 \\
\hline GW-786034 & FYN & 2700 & Sunitinib & SIK & 3200 \\
\hline GW-786034 & GAK & 200 & Sunitinib & skMLCK & 49 \\
\hline GW-786034 & HPK1 & 750 & Sunitinib & SLK & 56 \\
\hline GW-786034 & IRAK3 & 800 & Sunitinib & smMLCK & 280 \\
\hline GW-786034 & JAK3 & 6900 & Sunitinib & SNRK & 150 \\
\hline GW-786034 & JNK3 & 1900 & Sunitinib & SRC & 2100 \\
\hline GW-786034 & KDR & 14 & Sunitinib & SRPK1 & 250 \\
\hline GW-786034 & KHS1 & 3000 & Sunitinib & SRPK2 & 190 \\
\hline GW-786034 & KHS2 & 1600 & Sunitinib & STK33 & 17 \\
\hline GW-786034 & KIT & 2.8 & Sunitinib & TLK1 & 740 \\
\hline GW-786034 & LCK & 1200 & Sunitinib & TLK2 & 330 \\
\hline GW-786034 & LIMK1 & 720 & Sunitinib & TNIK & 25 \\
\hline GW-786034 & LIMK2 & 390 & Sunitinib & TNK1 & 680 \\
\hline GW-786034 & LOK & 84 & Sunitinib & TRKA & 100 \\
\hline GW-786034 & MAP2K4 & 590 & Sunitinib & TRKB & 590 \\
\hline GW-786034 & MAP2K6 & 4100 & Sunitinib & TRKC & 5100 \\
\hline GW-786034 & MARK3 & 4000 & Sunitinib & TSSK1 & 6300 \\
\hline GW-786034 & MER & 3300 & Sunitinib & TTK & 63 \\
\hline GW-786034 & MLK1 & 290 & Sunitinib & TYK2 & 1600 \\
\hline GW-786034 & MLK2 & 2100 & Sunitinib & TYRO3 & 49 \\
\hline GW-786034 & MLK3 & 740 & Sunitinib & Wee1 & 1100 \\
\hline GW-786034 & MPSK1 & 360 & Sunitinib & YES & 120 \\
\hline GW-786034 & NEK2 & 980 & Sunitinib & YSK1 & 290 \\
\hline GW-786034 & NLK & 4400 & VX-680/MK-0457 & AAK1 & 290 \\
\hline GW-786034 & PCTAIRE1 & 1200 & VX-680/MK-0457 & ABL1 & 13 \\
\hline GW-786034 & PDGFRa & 4.9 & VX-680/MK-0457 & ABL2 & 4 \\
\hline GW-786034 & PDGFRb & 2 & VX-680/MK-0457 & ACK & 4400 \\
\hline GW-786034 & PITSLRE & 2100 & VX-680/MK-0457 & ALK & 3100 \\
\hline GW-786034 & PITSLRE2 & 1300 & VX-680/MK-0457 & ALK2 & 1900 \\
\hline GW-786034 & PKR & 1900 & VX-680/MK-0457 & AMPKa1 & 1100 \\
\hline GW-786034 & PLK4 & 290 & VX-680/MK-0457 & AMPKa2 & 350 \\
\hline GW-786034 & QIK & 7300 & VX-680/MK-0457 & AurA & 4.1 \\
\hline GW-786034 & RAF1 & 900 & VX-680/MK-0457 & AurB & 7.4 \\
\hline GW-786034 & RET & 310 & VX-680/MK-0457 & AurC & 6.3 \\
\hline GW-786034 & RIPK1 & 260 & VX-680/MK-0457 & $A X L$ & 210 \\
\hline GW-786034 & RIPK2 & 580 & VX-680/MK-0457 & BIKE & 65 \\
\hline GW-786034 & ROS & 920 & VX-680/MK-0457 & BLK & 2200 \\
\hline GW-786034 & SIK & 2200 & VX-680/MK-0457 & BMPR2 & 7100 \\
\hline
\end{tabular}




\begin{tabular}{|c|c|c|c|c|c|}
\hline GW-786034 & skMLCK & 2000 & VX-680/MK-0457 & BMX & 2600 \\
\hline GW-786034 & SRC & 2800 & VX-680/MK-0457 & BTK & 4400 \\
\hline GW-786034 & SRM & 2500 & VX-680/MK-0457 & CaMK1d & 4900 \\
\hline GW-786034 & TIE1 & 700 & VX-680/MK-0457 & CaMLCK & 15 \\
\hline GW-786034 & TNIK & 310 & VX-680/MK-0457 & CK2a1 & 400 \\
\hline GW-786034 & TTK & 150 & VX-680/MK-0457 & CK2a2 & 400 \\
\hline GW-786034 & TXK & 2600 & VX-680/MK-0457 & CSK & 4800 \\
\hline GW-786034 & YES & 5000 & VX-680/MK-0457 & DCAMKL3 & 3600 \\
\hline Imatinib & ABL1 & 12 & VX-680/MK-0457 & DDR1 & 28 \\
\hline Imatinib & ABL2 & 10 & VX-680/MK-0457 & DDR2 & 230 \\
\hline Imatinib & BLK & 520 & VX-680/MK-0457 & DLK & 190 \\
\hline Imatinib & CDK11 & 5500 & VX-680/MK-0457 & EphA1 & 1000 \\
\hline Imatinib & CLK1 & 4500 & VX-680/MK-0457 & EphA2 & 840 \\
\hline Imatinib & CLK4 & 2100 & VX-680/MK-0457 & EphA3 & 1500 \\
\hline Imatinib & DDR1 & 0.7 & VX-680/MK-0457 & EphA4 & 4300 \\
\hline Imatinib & DDR2 & 15 & VX-680/MK-0457 & EphA8 & 500 \\
\hline Imatinib & DRAK1 & 5300 & VX-680/MK-0457 & EphB1 & 1900 \\
\hline Imatinib & EphA8 & 1400 & VX-680/MK-0457 & EphB4 & 1700 \\
\hline Imatinib & FGR & 2400 & VX-680/MK-0457 & FGFR1 & 550 \\
\hline Imatinib & FMS & 19 & VX-680/MK-0457 & FGFR2 & 570 \\
\hline Imatinib & FRK & 1500 & VX-680/MK-0457 & FGFR3 & 860 \\
\hline Imatinib & FYN & 3100 & VX-680/MK-0457 & FGFR4 & 7200 \\
\hline Imatinib & GAK & 1000 & VX-680/MK-0457 & FGR & 790 \\
\hline Imatinib & $\mathrm{HH} 498$ & 4300 & VX-680/MK-0457 & FLT1 & 100 \\
\hline Imatinib & JNK1 & 5000 & VX-680/MK-0457 & FLT3 & 6.5 \\
\hline Imatinib & JNK3 & 3100 & VX-680/MK-0457 & FLT4 & 1900 \\
\hline Imatinib & KIT & 14 & VX-680/MK-0457 & FMS & 1800 \\
\hline Imatinib & LCK & 40 & VX-680/MK-0457 & FRK & 3800 \\
\hline Imatinib & LYN & 890 & VX-680/MK-0457 & FYN & 530 \\
\hline Imatinib & MELK & 1900 & VX-680/MK-0457 & GAK & 3200 \\
\hline Imatinib & PDGFRa & 31 & VX-680/MK-0457 & GCN2 & 1900 \\
\hline Imatinib & PDGFRb & 14 & VX-680/MK-0457 & HGK & 2400 \\
\hline Imatinib & PLK4 & 7800 & VX-680/MK-0457 & HPK1 & 72 \\
\hline Imatinib & RAF1 & 1700 & VX-680/MK-0457 & IGF1R & 740 \\
\hline Imatinib & ZAK & 2600 & VX-680/MK-0457 & IKKe & 2600 \\
\hline JNJ-7706621 & AAK1 & 200 & VX-680/MK-0457 & INSR & 630 \\
\hline JNJ-7706621 & ABL1 & 1200 & VX-680/MK-0457 & IRAK3 & 5600 \\
\hline JNJ-7706621 & ACTR2 & 2900 & VX-680/MK-0457 & $\mathrm{IRR}$ & 290 \\
\hline JNJ-7706621 & ACTR2B & 5900 & VX-680/MK-0457 & ITK & 350 \\
\hline JNJ-7706621 & ADCK3 & 1200 & VX-680/MK-0457 & JAK2 & 190 \\
\hline JNJ-7706621 & ALK & 7100 & VX-680/MK-0457 & JAK3 & 630 \\
\hline JNJ-7706621 & ALK1 & 2900 & VX-680/MK-0457 & JNK3 & 2800 \\
\hline JNJ-7706621 & ALK2 & 5900 & VX-680/MK-0457 & KDR & 2000 \\
\hline JNJ-7706621 & AMPKa1 & 1700 & VX-680/MK-0457 & $\mathrm{KHS1}$ & 83 \\
\hline
\end{tabular}




\begin{tabular}{|c|c|c|c|c|c|}
\hline JNJ-7706621 & AMPKa2 & 1800 & VX-680/MK-0457 & KHS2 & 290 \\
\hline JNJ-7706621 & AurA & 250 & VX-680/MK-0457 & KIT & 240 \\
\hline JNJ-7706621 & AurB & 160 & VX-680/MK-0457 & LATS1 & 5500 \\
\hline JNJ-7706621 & AurC & 86 & VX-680/MK-0457 & LCK & 61 \\
\hline JNJ-7706621 & $A X L$ & 2200 & VX-680/MK-0457 & LIMK1 & 2600 \\
\hline JNJ-7706621 & BIKE & 180 & VX-680/MK-0457 & LIMK2 & 2600 \\
\hline JNJ-7706621 & BMPR2 & 880 & VX-680/MK-0457 & LKB1 & 1300 \\
\hline JNJ-7706621 & $\mathrm{BMX}$ & 4700 & VX-680/MK-0457 & LOK & 120 \\
\hline JNJ-7706621 & BRSK1 & 6500 & VX-680/MK-0457 & LTK & 7500 \\
\hline JNJ-7706621 & BRSK2 & 2700 & VX-680/MK-0457 & LYN & 650 \\
\hline JNJ-7706621 & CaMKK1 & 2900 & VX-680/MK-0457 & MAP2K1 & 1800 \\
\hline JNJ-7706621 & CaMKK2 & 2600 & VX-680/MK-0457 & MAP2K2 & 2000 \\
\hline JNJ-7706621 & CDK2 & 23 & VX-680/MK-0457 & MAP2K6 & 2600 \\
\hline JNJ-7706621 & CDK3 & 180 & VX-680/MK-0457 & MAP3K4 & 2500 \\
\hline JNJ-7706621 & CDK5 & 240 & VX-680/MK-0457 & MARK1 & 4900 \\
\hline JNJ-7706621 & CDK7 & 760 & VX-680/MK-0457 & MARK2 & 1400 \\
\hline JNJ-7706621 & CDK9 & 470 & VX-680/MK-0457 & MARK3 & 2500 \\
\hline JNJ-7706621 & CK1e & 3600 & VX-680/MK-0457 & MARK4 & 4900 \\
\hline JNJ-7706621 & CK1g1 & 1200 & VX-680/MK-0457 & MELK & 240 \\
\hline JNJ-7706621 & CK1g2 & 740 & VX-680/MK-0457 & MER & 1700 \\
\hline JNJ-7706621 & CK1g3 & 7900 & VX-680/MK-0457 & MET & 670 \\
\hline JNJ-7706621 & CK2a2 & 2000 & VX-680/MK-0457 & MLK1 & 390 \\
\hline JNJ-7706621 & CLK1 & 660 & VX-680/MK-0457 & MLK2 & 1800 \\
\hline JNJ-7706621 & CLK2 & 290 & VX-680/MK-0457 & MLK3 & 680 \\
\hline JNJ-7706621 & CLK3 & 310 & VX-680/MK-0457 & MPSK1 & 610 \\
\hline JNJ-7706621 & CLK4 & 1700 & VX-680/MK-0457 & MST1 & 200 \\
\hline JNJ-7706621 & DCAMKL1 & 2500 & VX-680/MK-0457 & MST2 & 510 \\
\hline JNJ-7706621 & DCAMKL2 & 4500 & VX-680/MK-0457 & MST3 & 3600 \\
\hline JNJ-7706621 & DCAMKL3 & 6500 & VX-680/MK-0457 & MUSK & 300 \\
\hline JNJ-7706621 & DLK & 5200 & VX-680/MK-0457 & NEK5 & 4200 \\
\hline JNJ-7706621 & DMPK1 & 1200 & VX-680/MK-0457 & NuaK1 & 200 \\
\hline JNJ-7706621 & DRAK1 & 5600 & VX-680/MK-0457 & PAK1 & 4400 \\
\hline JNJ-7706621 & EphA7 & 3100 & VX-680/MK-0457 & PAK2 & 5700 \\
\hline JNJ-7706621 & ErK3 & 480 & VX-680/MK-0457 & PAK3 & 3300 \\
\hline JNJ-7706621 & ErK4 & 1600 & VX-680/MK-0457 & PAK4 & 4300 \\
\hline JNJ-7706621 & ErK5 & 520 & VX-680/MK-0457 & PAK5 & 3300 \\
\hline JNJ-7706621 & Erk7 & 1100 & VX-680/MK-0457 & PCTAIRE1 & 230 \\
\hline JNJ-7706621 & FGFR1 & 810 & VX-680/MK-0457 & PDGFRa & 1600 \\
\hline JNJ-7706621 & FGFR2 & 680 & VX-680/MK-0457 & PDGFRb & 310 \\
\hline JNJ-7706621 & FGFR3 & 510 & VX-680/MK-0457 & PHKg1 & 2200 \\
\hline JNJ-7706621 & FGR & 2000 & VX-680/MK-0457 & PIP5K1A & 130 \\
\hline JNJ-7706621 & FLT1 & 2100 & VX-680/MK-0457 & PIP5K2B & 140 \\
\hline JNJ-7706621 & FLT3 & 850 & VX-680/MK-0457 & PITSLRE & 290 \\
\hline JNJ-7706621 & FLT4 & 1100 & VX-680/MK-0457 & PITSLRE2 & 260 \\
\hline
\end{tabular}




\begin{tabular}{|c|c|c|c|c|c|}
\hline JNJ-7706621 & FMS & 4200 & VX-680/MK-0457 & PKN1 & 1500 \\
\hline JNJ-7706621 & GAK & 2400 & VX-680/MK-0457 & PLK4 & 9.2 \\
\hline JNJ-7706621 & GCN2 & 3900 & VX-680/MK-0457 & PYK2 & 2600 \\
\hline JNJ-7706621 & GSK3A & 160 & VX-680/MK-0457 & QIK & 1700 \\
\hline JNJ-7706621 & GSK3B & 950 & VX-680/MK-0457 & RET & 28 \\
\hline JNJ-7706621 & $\mathrm{HH} 498$ & 1400 & VX-680/MK-0457 & RIOK1 & 360 \\
\hline JNJ-7706621 & HPK1 & 120 & VX-680/MK-0457 & RIOK3 & 600 \\
\hline JNJ-7706621 & IKKe & 8300 & VX-680/MK-0457 & RIPK1 & 20 \\
\hline JNJ-7706621 & IRAK3 & 3400 & VX-680/MK-0457 & ROS & 610 \\
\hline JNJ-7706621 & JAK2 & 220 & VX-680/MK-0457 & SgK085 & 2200 \\
\hline JNJ-7706621 & JAK3 & 180 & VX-680/MK-0457 & SgK288 & 5600 \\
\hline JNJ-7706621 & JNK1 & 1700 & VX-680/MK-0457 & SIK & 2500 \\
\hline JNJ-7706621 & JNK3 & 1300 & VX-680/MK-0457 & skMLCK & 43 \\
\hline JNJ-7706621 & KDR & 3700 & VX-680/MK-0457 & SLK & 82 \\
\hline JNJ-7706621 & KHS1 & 2100 & VX-680/MK-0457 & SNRK & 530 \\
\hline JNJ-7706621 & KHS2 & 820 & VX-680/MK-0457 & SRC & 170 \\
\hline JNJ-7706621 & KIT & 1800 & VX-680/MK-0457 & SRPK1 & 82 \\
\hline JNJ-7706621 & LIMK1 & 620 & VX-680/MK-0457 & SRPK2 & 680 \\
\hline JNJ-7706621 & LIMK2 & 1000 & VX-680/MK-0457 & SYK & 1800 \\
\hline JNJ-7706621 & LOK & 110 & VX-680/MK-0457 & TESK1 & 5600 \\
\hline JNJ-7706621 & MAP2K1 & 1800 & VX-680/MK-0457 & TIE1 & 270 \\
\hline JNJ-7706621 & MAP2K2 & 5600 & VX-680/MK-0457 & TIE2 & 450 \\
\hline JNJ-7706621 & MAP2K3 & 1500 & VX-680/MK-0457 & TNIK & 1800 \\
\hline JNJ-7706621 & MAP2K4 & 630 & VX-680/MK-0457 & TNK1 & 83 \\
\hline JNJ-7706621 & MAP2K6 & 1000 & VX-680/MK-0457 & TRKA & 38 \\
\hline JNJ-7706621 & MARK1 & 620 & VX-680/MK-0457 & TRKB & 240 \\
\hline JNJ-7706621 & MARK2 & 1200 & VX-680/MK-0457 & TRKC & 200 \\
\hline JNJ-7706621 & MARK3 & 600 & VX-680/MK-0457 & TXK & 2700 \\
\hline JNJ-7706621 & MARK4 & 660 & VX-680/MK-0457 & Wee1 & 3200 \\
\hline JNJ-7706621 & MER & 2800 & VX-680/MK-0457 & YES & 470 \\
\hline JNJ-7706621 & MET & 650 & VX-680/MK-0457 & YSK1 & 4800 \\
\hline JNJ-7706621 & MLK1 & 3600 & VX-680/MK-0457 & ZAK & 2800 \\
\hline JNJ-7706621 & MPSK1 & 140 & $V X-745$ & ABL1 & 730 \\
\hline JNJ-7706621 & MST1 & 130 & VX-745 & ABL2 & 1900 \\
\hline JNJ-7706621 & MST2 & 61 & VX-745 & BLK & 3100 \\
\hline JNJ-7706621 & MST3 & 180 & VX-745 & DDR1 & 1100 \\
\hline JNJ-7706621 & MST4 & 210 & VX-745 & FGR & 1300 \\
\hline JNJ-7706621 & NuaK1 & 180 & VX-745 & FMS & 2600 \\
\hline JNJ-7706621 & PAK3 & 2100 & VX-745 & FYN & 2100 \\
\hline JNJ-7706621 & PAK4 & 2300 & VX-745 & LCK & 4600 \\
\hline JNJ-7706621 & PAK5 & 830 & VX-745 & LYN & 1700 \\
\hline JNJ-7706621 & PAK6 & 1400 & VX-745 & p38a & 2.8 \\
\hline JNJ-7706621 & PCTAIRE1 & 23 & VX-745 & p38b & 74 \\
\hline JNJ-7706621 & PCTAIRE2 & 290 & VX-745 & PDGFRb & 8400 \\
\hline
\end{tabular}




\begin{tabular}{|c|c|c|c|c|c|}
\hline JNJ-7706621 & PCTAIRE3 & 2100 & VX-745 & SRC & 5500 \\
\hline JNJ-7706621 & PDGFRa & 4000 & VX-745 & YES & 1600 \\
\hline JNJ-7706621 & PDGFRb & 910 & ZD-6474 & ABL1 & 270 \\
\hline JNJ-7706621 & PDK1 & 3100 & ZD-6474 & ABL2 & 69 \\
\hline JNJ-7706621 & PFTAIRE1 & 3600 & ZD-6474 & ADCK3 & 4500 \\
\hline JNJ-7706621 & PIP5K1A & 9200 & ZD-6474 & ADCK4 & 1700 \\
\hline JNJ-7706621 & PIP5K2B & 1300 & ZD-6474 & ALK & 2100 \\
\hline JNJ-7706621 & PITSLRE & 150 & ZD-6474 & ALK1 & 470 \\
\hline JNJ-7706621 & PITSLRE2 & 110 & ZD-6474 & ALK2 & 150 \\
\hline JNJ-7706621 & PKCd & 4500 & ZD-6474 & AurC & 1500 \\
\hline JNJ-7706621 & PKCt & 4400 & ZD-6474 & $A X L$ & 250 \\
\hline JNJ-7706621 & PKD1 & 8400 & ZD-6474 & BLK & 66 \\
\hline JNJ-7706621 & PKD3 & 4200 & ZD-6474 & BRK & 160 \\
\hline JNJ-7706621 & PKN2 & 1900 & ZD-6474 & CK1e & 3000 \\
\hline JNJ-7706621 & PKR & 6300 & ZD-6474 & CRIK & 1800 \\
\hline JNJ-7706621 & PLK1 & 5000 & ZD-6474 & CSK & 2500 \\
\hline JNJ-7706621 & PLK3 & 7100 & ZD-6474 & DDR1 & 11 \\
\hline JNJ-7706621 & PLK4 & 120 & ZD-6474 & DDR2 & 320 \\
\hline JNJ-7706621 & QIK & 1600 & ZD-6474 & DMPK2 & 2200 \\
\hline JNJ-7706621 & RET & 1000 & ZD-6474 & EGFR & 9.5 \\
\hline JNJ-7706621 & RIOK1 & 1100 & ZD-6474 & EphA1 & 230 \\
\hline JNJ-7706621 & RIOK3 & 1200 & ZD-6474 & EphA2 & 1100 \\
\hline JNJ-7706621 & RIPK1 & 1800 & ZD-6474 & EphA3 & 2000 \\
\hline JNJ-7706621 & SgK288 & 500 & ZD-6474 & EphA4 & 1600 \\
\hline JNJ-7706621 & SIK & 2200 & ZD-6474 & EphA5 & 240 \\
\hline JNJ-7706621 & SLK & 48 & ZD-6474 & EphA6 & 50 \\
\hline JNJ-7706621 & smMLCK & 2100 & ZD-6474 & EphA7 & 2400 \\
\hline JNJ-7706621 & SNRK & 26 & ZD-6474 & EphA8 & 91 \\
\hline JNJ-7706621 & SRPK1 & 2800 & ZD-6474 & EphB1 & 290 \\
\hline JNJ-7706621 & SYK & 1300 & ZD-6474 & EphB2 & 440 \\
\hline JNJ-7706621 & TEC & 1000 & ZD-6474 & EphB4 & 520 \\
\hline JNJ-7706621 & TESK1 & 3100 & ZD-6474 & ErbB2 & 2600 \\
\hline JNJ-7706621 & TIE1 & 260 & ZD-6474 & ErbB4 & 480 \\
\hline JNJ-7706621 & TIE2 & 950 & ZD-6474 & ErK3 & 1500 \\
\hline JNJ-7706621 & TLK1 & 1100 & ZD-6474 & FGFR1 & 560 \\
\hline JNJ-7706621 & TLK2 & 750 & ZD-6474 & FGFR2 & 1100 \\
\hline JNJ-7706621 & TNK1 & 980 & ZD-6474 & FGFR3 & 1600 \\
\hline JNJ-7706621 & TTK & 190 & ZD-6474 & FGR & 270 \\
\hline JNJ-7706621 & TYK2 & 32 & ZD-6474 & FLT1 & 260 \\
\hline JNJ-7706621 & YSK1 & 360 & ZD-6474 & FLT3 & 850 \\
\hline Lapatinib & EGFR & 2.4 & ZD-6474 & FLT4 & 1100 \\
\hline Lapatinib & ErbB2 & 7 & ZD-6474 & FMS & 1200 \\
\hline Lapatinib & ErbB4 & 54 & ZD-6474 & FRK & 170 \\
\hline Lapatinib & LOK & 4400 & ZD-6474 & FYN & 360 \\
\hline
\end{tabular}




\begin{tabular}{|c|c|c|c|c|c|}
\hline Lapatinib & RIPK2 & 3600 & ZD-6474 & GAK & 86 \\
\hline LY-333531 & AAK1 & 900 & ZD-6474 & HCK & 360 \\
\hline LY-333531 & AKT3 & 1400 & ZD-6474 & HGK & 1400 \\
\hline LY-333531 & BIKE & 430 & ZD-6474 & HH498 & 2800 \\
\hline LY-333531 & CaMK2a & 4500 & ZD-6474 & KDR & 820 \\
\hline LY-333531 & CaMK2d & 4600 & ZD-6474 & KHS1 & 450 \\
\hline LY-333531 & CaMK2g & 7800 & ZD-6474 & KHS2 & 1500 \\
\hline LY-333531 & CaMKK1 & 5000 & ZD-6474 & KIT & 260 \\
\hline LY-333531 & CaMKK2 & 2400 & ZD-6474 & LCK & 17 \\
\hline LY-333531 & $\mathrm{CHK} 1$ & 540 & ZD-6474 & LOK & 81 \\
\hline LY-333531 & CK2a1 & 3600 & ZD-6474 & LTK & 550 \\
\hline LY-333531 & CK2a2 & 3400 & ZD-6474 & LYN & 110 \\
\hline LY-333531 & CLK1 & 910 & ZD-6474 & MAP2K1 & 1800 \\
\hline LY-333531 & CLK2 & 420 & ZD-6474 & MAP2K2 & 1100 \\
\hline LY-333531 & CLK4 & 1900 & ZD-6474 & MER & 1400 \\
\hline LY-333531 & CRIK & 1400 & ZD-6474 & MET & 5700 \\
\hline LY-333531 & DAPK1 & 970 & ZD-6474 & MNK1 & 360 \\
\hline LY-333531 & DMPK1 & 280 & ZD-6474 & MNK2 & 1700 \\
\hline LY-333531 & DYRK1B & 5400 & ZD-6474 & MRCKa & 2600 \\
\hline LY-333531 & Erk7 & 88 & ZD-6474 & MRCKb & 2500 \\
\hline LY-333531 & FLT3 & 1900 & ZD-6474 & PDGFRa & 230 \\
\hline LY-333531 & GAK & 1100 & ZD-6474 & PDGFRb & 88 \\
\hline LY-333531 & GSK3B & 2600 & ZD-6474 & PHKg1 & 1100 \\
\hline LY-333531 & HPK1 & 2600 & ZD-6474 & PLK4 & 620 \\
\hline LY-333531 & JAK3 & 420 & ZD-6474 & QIK & 430 \\
\hline LY-333531 & KHS2 & 8400 & ZD-6474 & RET & 34 \\
\hline LY-333531 & LATS1 & 2600 & ZD-6474 & RIPK2 & 4.6 \\
\hline LY-333531 & LATS2 & 640 & ZD-6474 & SIK & 1900 \\
\hline LY-333531 & LOK & 280 & ZD-6474 & SLK & 95 \\
\hline LY-333531 & MAP2K4 & 930 & ZD-6474 & SRC & 70 \\
\hline LY-333531 & MLK1 & 1400 & ZD-6474 & SRM & 1900 \\
\hline LY-333531 & MST2 & 960 & ZD-6474 & STK33 & 1400 \\
\hline LY-333531 & NuaK1 & 1800 & ZD-6474 & TESK1 & 3700 \\
\hline LY-333531 & PDK1 & 700 & ZD-6474 & TIE1 & 1500 \\
\hline LY-333531 & PHKg1 & 3700 & ZD-6474 & TIE2 & 1000 \\
\hline LY-333531 & PIM1 & 270 & ZD-6474 & TNIK & 2300 \\
\hline LY-333531 & PIM2 & 1700 & ZD-6474 & TXK & 3700 \\
\hline LY-333531 & PIM3 & 12 & ZD-6474 & TYRO3 & 93 \\
\hline LY-333531 & PIP5K1A & 690 & ZD-6474 & YES & 120 \\
\hline LY-333531 & PIP5K2B & 830 & ZD-6474 & ZAK & 5100 \\
\hline
\end{tabular}

